\title{
Los macroinvertebrados como bioindicadores de la calidad del agua: cuatro décadas de desarrollo en Colombia y Latinoamerica
}

\author{
Gabriel Roldán-Pérez
}

Grupo de Investigación de Limnología y Recursos Hídricos, Universidad Católica de Oriente, Rionegro, Colombia

Artículo de posesión para el ingreso como miembro de número a la

Academia Colombiana de Ciencias Exactas, Físicas y Naturales el 6 de abril de 2016

\begin{abstract}
Resumen
La comunidad de macroinvertebrados acuáticos mejor estudiada en Colombia es la entomofauna. Los órdenes Ephemeroptera, Plecoptera y Trichoptera son los mejor conocidos en cuanto a su taxonomía, ecología y su utilización como bioindicadores de la calidad del agua. Es necesario profundizar en el estudio de algunos grupos como los anélidos, moluscos, ácaros y dípteros, de los cuales aun falta conocimiento de su taxonomía y autoecología. El índice BMWP (Biological Monitoring Working Party), desarrollado en Europa en el siglo XX a partir de la década de los años setenta, es muy popular en Colombia y Latinoamérica, pero es necesario hacer adaptaciones para las diferentes regiones en el continente.Este trabajo presenta una revisión de los estudios realizados durante las últimas cuatro décadas en Colombia, y de manera general en Latinoamérica. Se presenta el estado actual del conocimiento de los diferentes grupos de los macroinvertebrados acuáticos en términos de la resolución taxonómica, los aspectos ecológicos y su utilización como bioindicadores de la calidad del agua. ㄷ Acad. Colomb. Cienc. Ex. Fis. Nat. 2016.
\end{abstract}

Palabras clave: macroinvertebrados, bioindicacion, índice BMWP, Colombia.

Macroinvertebrates as bioindicators of water quality: four decades of development in Colombia and Latin America

\begin{abstract}
Entomofauna is the community of aquatic macroinvertebrates best studied in Colombia. Ephemeroptera, Trichoptera, and Plecoptera orders are the better known in the taxonomy, ecology and their use as bioindicators of water quality. It is necessary to deepen the study of some groups such as annelids, molluscs, mites and dipterans, which still lack knowledge of their taxonomy and autoecology. The BMWP (Biological Monitoring Working Party) index, developed in Europe in the twentieth century in the early seventies, is very popular in Colombia and Latin America, but it is needed to make more detailed adjustments for different regions on the continent. This paper presents a review of studies conducted during the last four decades in Colombia in particular, and more generally, in Latin America. The current state of knowledge of the different groups of aquatic macroinvertebrates in terms of taxonomic resolution, environmental aspects and its use as bioindicators of water quality analyzes is presented. (c) Acad. Colomb. Cienc. Ex. Fis. Nat. 2016.
\end{abstract}

Key words: Macroinvertebrates, bioindication, BMWP index, Colombia.

\section{Introducción}

Los macroinvertebrados acuáticos son todos aquellos organismos que viven en el fondo de ríos y lagos, adheridos a la vegetación acuática, troncos y rocas sumergidas. Sus poblaciones están conformadas por platelmintos, insectos, moluscos y crustáceos principalmente. Se les denomina macroinvertebrados, porque su tamaño va de $0.5 \mathrm{~mm}$ hasta alrededor de $5.0 \mathrm{~mm}$, por lo que se les puede observar a simple vista. Es un hecho que la composición de las comunidades de macroinvertebrados refleja la calidad de los ecosistemas acuáticos; por ello, los métodos de evaluación basados en dichos organismos han sido ampliamente utilizados desde hace varias décadas como una parte integral del monitoreo de la calidad del agua. Los países de la Unión Europea y Norte América han sido los líderes en este proceso (Gaufin y
Tarzwell, 1952; Hynes, 1959; Resh et al. 1995. Los estudios basados en esta metodología han permitido un conocimiento del estado ecológico de los ríos y lagos europeos, lo cual sirvió de base para lograr una sorprendente recuperación de éstos ecosistemas en los últimos 20 años.

De acuerdo con el conocimiento que se tenga de la fauna acuática en cada país, esta evaluación podrá hacerse en diferentes niveles de precisión. Los indices son una de las formas numéricas biológicas que generan informacion y criterios para la evaluación de la contaminacion, basados en

\footnotetext{
Correspondencia:

Gabriel Roldán-Pérez, groldan@uco.edu.co

Recibido: 9 de febrero de 2016
}

Aceptado: 25 de abril de 2016 
la integridad ecológica. Estos índices pueden ser unimétricos, multimétricos, multivariados y de rasgos biológicos. Los índices multimétricos combinan en una puntuacién final el valor independiente de diversas métricas, que pueden ser simples o indices bióticos. Alemania que ha tenido una tradición más larga en el conocimiento de su flora y fauna acuática, ha adoptado el método saprobio, el cual requiere para su aplicación la identificación de los organismos hasta el nivel de especie. Otros países, como Bélgica, Francia, Gran Bretaña, Italia, Portugal, Dinamarca, Holanda e Irlanda, han adoptado sistemas de evaluación basados en el nivel de ordenes, familias y en algunos casos de géneros. Para la denominada “evaluación rápida del ecosistema”, se ha comprobado su efectividad en un alto porcentaje, además de una considerable reducción de costos y de tiempo (De Pauw y Hawkes, 1993; Roldán, 2003). Statzner et al. (2001) discuten las perspectivas para el biomonitoreo en escalas espaciales como una unidad de medida de la composición funcional de las comunidades de macroinvertebrados. Mendes et al. (2014) proponen combinar el uso de las diatomeas con los macroinvertebrados en la evaluación de la calidad del agua.

La evaluación de la calidad del agua se ha realizado tradicionalmente basada en los análisis fisicoquímicos y bacteriológicos. Sin embargo, en los últimos años, muchos países han aceptado la inclusión de los macroinvertebrados para evaluar la calidad de los ecosistemas acuáticos. Para el ecólogo, un ecosistema acuático es un sistema funcional en el cual hay un intercambio cíclico de materia y energía entre los organismos vivos y el ambiente abiótico. Por lo tanto, la biología y la química, están estrechamente relacionadas; en la evaluación de las aguas naturales y contaminadas juegan papeles complementarios.

\section{Desarrollo histórico de la bioindicacion de la calidad del agua}

La utilización de los organismos acuáticos como bioindicadores de la calidad de los ecosistemas acuáticos comienza en Europa mediados del siglo XIX. Kolenati (1848) y Cohn (1853), encontraron relaciones entre ciertas especies y el grado de calidad del agua. En el siglo XX, Kolkowitz \& Marsson (1908, 1909) propusieron el sistema saprobio para Alemania, que actualmente es adoptado en otros países europeos. Patrick (1949, 1950) desarrolla métodos biológicos para evaluar las condiciones ecológicas de las corrientes en Norteamérica y Gaufin y Tarzwell (1952) proponen los macroinvertebrados como indicadores de contaminación. El sistema limnosaprobio es introducido en Checoeslovaquia por Sládeček (1962). Hynes $(1959,1963)$ propone los macroinvertebrados como indicadores de la calidad del agua. Illies y Botosaneau (1963) discuten la zonación de las aguas corrientes y proponen los términos de ritrón y potamón para referirse a las zonas altas y bajas de los ríos; este trabajo se considera un clásico de la limnología europea.
El concepto de diversidad de especies basado en índices matemáticos derivados de la teoría de la información, comienza a aplicarse en los años cincuenta y sesenta. (Shannon \& Weaver, 1949; Brillouin, 1951; Margalef, 1951; Beck, 1955; Wilhm \& Dorris, 1966; 1968; y Wilhm, 1967, 1968). Dicha teoría parte de la base, que mientras mayor información se tenga acerca de un hecho, suceso o situación, mayor y más preciso será el entendimiento que se tenga de él. Washington (1984) hace una revisión de los índices de diversidad, bióticos y de similitud con especial referencia a los ecosistemas acuáticos. Presenta18 índices de diversidad, 19 índices bióticos y cinco índices de similitud y analizó su aplicabilidad a los sistemas biológicos.

Resh et al. (1995) desarrollaron en Maryland (Estados Unidos) métodos rápidos de evaluación de la calidad del agua usando los macroinvertebrados como bioindicadores. Tanto este método como el del Reino Unido valoran las condiciones del hábitat y predicen la fauna esperada en un determinado sitio. Trihadiningrum et al. (1996) utilizaron los macroinvertebrados como indicadores de la calidad del agua en Indonesia. Alba-Tercedor (1996, 1988) adoptó la utilización de los macroinvertebrados acuáticos en los programas de evaluación de la calidad del agua en España, utilizando para ello el índice BMWP’ (Biological Monitoring Water Party) adaptado para la Península Ibérica. Towsand \& Scarsbrook (1997) calificaron la perturbación en las corrientes en relación con las características de los macroinvertebrados y la riqueza de dichas especies.

Lorenz, et al., (1997) desarrollan un sistema de bioindicadores en el río Rin (Alemania) con base en conceptos teóricos que describen los ríos naturales, entre los cuales se consideran la zonación, la hidráulica, el espiral de nutrientes, la jerarquía de tributarios y el concepto de río continuo, entre otros. Munné et al. 1998) proponen un índice de calidad en España que valora el estado de calidad de conservación del bosque de ribera (QBR). De Pauw \& Hawkes (1993) analizan el desarrollo de la bioidicación en la Unión Europea a partir de la década de los años setenta y en especial de los noventa. La tabla 1 muestra la aplicación de los principales métodos para la evaluación de la calidad del agua en la Unión Europea basada en los macroinvertebrados acuáticos. Nótese la simplicidad de los diferentes métodos, los cuales son cualitativos y cómo la identificación de los organismos sólo llega hasta el nivel de familia en la mayoría de los casos. Es importante hacer énfasis que el método consiste en una evaluación rápida del ecosistema y que en ningún momento pretende definir los requerimientos ambientales a nivel de especie.

Birk \& Herring (2006) hacen una comparación directa de los métodos de valoración usando los macroinvertebrados como bioindicadores. Carsten et al. (2007) discuten los índices de calidad del agua en Europa como indicador de un buen estado ecológico. Tambien existe el Indice de Integridad Biótica (IIB o IBI) o Indice de Integridad Biológica, el cual es una herramienta multimétrica para identificar y clasificar los problemas de contaminación del agua. 
Tabla 1. Aplicación de los principales métodos de bioindicación para la evaluación de las aguas corrientes en los países de la Unión Europea con base en los macroinvertebrados (De Pauw y Hawkes, 1993).

\begin{tabular}{|c|c|c|c|c|c|c|}
\hline País & Método de indicación & Muestreo & Análisis & Identificación $^{1}$ & Estándar ${ }^{2}$ & Rango \\
\hline Bélgica & B B I & Cualitativo & Cualitativo & O F G & $\mathrm{N}$ & $0-10$ \\
\hline Dinamarca & D F I & Cualitativo & Cualitativo & F G S & $\mathrm{N}$ & $1-4$ \\
\hline Francia & I B G N & Cuant/Cual & Cualitativo & F & $\mathrm{N}$ & $0-20$ \\
\hline Alemania & B E O L / S & Cualitativo & Cuantitativo & S & $\mathrm{N}$ & $0-100 / 1-4$ \\
\hline Irlanda & $\mathrm{Q}$ - rating & Cualitativo & Cualitativo & F G S & $\mathrm{N}$ & $0-5$ \\
\hline Italia & E B I & Cualitativo & Cualitativo & O F G & $\mathrm{R}$ & $0-14$ \\
\hline Luxemburgo & I B & Cualitativo & Cualitativo & O F & $\mathrm{N}$ & $0-10$ \\
\hline Holanda & K 135 & Cualitativo & Cualitativo & F G S & $\mathrm{R}$ & $100-500$ \\
\hline Portugal & B B I & Cualitativo & Cualitativo & O F G & - & $0-10$ \\
\hline España & BMWP' & Cualitativo & Cualitativo & $\mathrm{F}$ & - & $0->150$ \\
\hline Reino Unido & BMWP/ASPT & Cualitativo & Cualitativo & $\mathrm{F}$ & $\mathrm{N}$ & $0->150 / 0-10$ \\
\hline
\end{tabular}

$\mathrm{O}=$ Orden, $\mathrm{F}=$ Familia; $\mathrm{G}=$ Género; $\mathrm{S}=$ Especie $\quad 2 . \mathrm{N}=$ Nacional, $\mathrm{R}=$ Regional

\section{Estado actual del conocimiento sobre los macroinvertebrados acuáticos en Colombia}

Los primeros estudios de macroinvertebrados acuáticos en Colombia se iniciaron en la década de los años setenta (Roldán et al.1973; Pérez \& Roldán, 1978). Las identificaciones preliminares se realizaron con base en las claves disponibles en esa época, desarrolladas en su mayoría por especialistas norteamericanos y europeos. Una vez verificado el primer reporte con especialistas, se encontró que la mayoría del material estaba incorrectamente identificado, pues las claves de estos autores estaban hechas para zonas templadas. Era por lo tanto, necesario elaborar claves con base en las recolectas hechas en el territorio colombiano.

Al inicio de la década de 1980 y con el apoyo de entomólogos americanos, se comenzó la elaboración de claves taxonómicas para cada uno de los grupos de macroinvertebrados acuáticos presentes en Colombia. En este proyecto participaron estudiantes del Programa de Biología en la Universidad de Antioquia, quienes aportaron a través de sus trabajos de pregrado valiosa información al conocimiento de los diferentes órdenes de la comunidad de macroinvertebrados acuáticos. Como producto de estos estudios Roldán (1988) publicó la "Guía para el Estudio de los Macroinvertebrados Acuáticos del Departamento de Antioquia”, trabajo que sirvió de referencia para el inicio del conocimiento de las comunidadesde estos organismos en diferentes regiones de Colombia y de Latinoamérica.

\section{Estudios taxonómicos}

El conocimiento de la taxonomía de los macroinvertebrados en los ecosistemas acuáticos es todavía incompleto en Colombia. Los grupos mas utilizados en evaluaciones de la calidad del agua corresponden a taxones de la entomofauna como efemerópteros, tricópteros, plecópteros y coleópteros. Perla et al. (2014) llevan acabo un extenso análisis del estado de conocimiento de los macroinvertebrdos acuáticos dulceacuícolas de México, Centroamérica, Colombia, Cuba y Puerto Rico. Roldán et al. (2014) escriben en esta publicación el Capitulo para Colombia, cuyo contenido es hasta el presente el documentado más completo para nuestro país. A continución se hace una revisión del estado actual de cada uno de los diferentes taxones.

\section{Phylum Platyhelmintes}

Los primeros reportes de los turbelarios de agua dulce en Colombia fueron hechas por Fuhrmann (1914), Furmann \& Mayor (1914) y Ball (1969, 1980). El género Dugesia (Planariidae) es reportado para los ríos Medellín y Rionegro en aguas poco contaminadas (Roldán et al. 1973, Pérez \& Roldán 1978). Muñoz \& Vélez (2007) reportaron en 22 sistemas acuáticos del centro y sur oriente del departamento de Antioquia las especies Girardia cameliae, G. paramensis y G. tigrina (Dugesiidae).

\section{Phylum Annelida}

La subclase Hirudinea es un grupo que se encuentra principalmente en ambientes acuáticos degradados, ricos en carga orgánica residual y baja disponibidad de oxígeno disuelto. Los primeros reportes de hirudíneos colombianos en ecosistemas acuáticos de diferentes regiones, incluida la zona altoandina de los páramos, fueron hechos por Weber (1913), Michaelsen (1913-1914) y Ringuelet (1972, 1974, 1975). Gaviria (1993) elabora una clave para las familias Naidae y Tubificidae (Tubifex) presentes en Colombia.

\section{Phylum Arthropoda}

\section{Clase Insecta}

Orden Ephemeroptera. Los primeros estudios en Colombia se realizaron en el departamento de Antioquia, con énfasis en su taxonomía y distribución en diferentes gradientes 
altitudinales (Roldán, 1980, 1985, 1988). Posteriormente, diferentes autores contribuyeron en al avance del conocimiento del orden en varias regiones del país, mediante el registro y descripción de nuevos géneros y especies, particularmente de las familias Leptohyphidae, Baetidae, Leptophlebiidae y Polymitarciydae (Muñoz \& Ospina, 1999; Muñoz, 2000; Mosquera et al. 2001; Domínguez et al. 2002, 2009; Domínguez \& Zúñiga, 2003, 2009; Camargo \& Rozo, 2003; Molineri, 2010; Molineri et al. 2002, 2011; Molineri \& Zúñiga, 2004, 2006; Gutiérrez \& Reinoso 2010; Salinas et al.2011; Días et al. 2011; Forero et al. 2013 y Forero \& Reinoso, 2013).

El Orden Ephemeroptera contiene las familias Baetidae, Caenidae, Coryphoridae, Ephemeridae, Euthyplociidae, Leptohyphidae, Leptophlebiidae, Oligoneuriidae y Polymitarcyidae. Las familias Baetidae, Leptophlebiidae y Leptohyphidae son las de mayor distribución y diversidad y la región natural andina es la que cuenta con el mayor número de registros específicos (Roldán, 1988; Reinoso, 1999; Zúñiga, et al. 2004, Gutiérrez \& Reinoso, 2010). A nivel sudamericano se encuentra disponible una publicación que recopila la información sobre las ninfas y los imagos del orden en la región y que incluye aspectos taxonómicos, ecológicos y de distribución (Domínguez \& Fernández, 2009).

Orden Odonata. Los primeros reportes en el país fueron hechos por Williamson (1918a, b, 1919, 1920); Navas, 1935 y Hincks, 1934). Trabajos sobre la taxonomía, la riqueza de este orden y su distribución en algunas regiones como Antioquia, distrito de Santa Marta, Valle del Cauca, Boyacá y las zonas del pacífico y el atlántico, fueron realizados por Arango \& Roldán, (1983); Cruz, (1986); Suárez, (1987); De Marmels (1982a,b, 2001); NoveloGutiérrez (1995a,b); Ramírez (1996); Ramírez \& NoveloGutiérrez (1999); Pérez (2003); Ceballos (2004); Astudillo (2005); Bermúdez (2005), Palacino-Rodríguez (2009); Urrutia (2005); Altamiranda-Saavedra et al. (2010) y Garzón \& Realpe (2009). Rodríguez (2009), evaluó los anisopteros depositados en la Colección del Instituto de Ciencias Naturales de la Universidad Nacional de Colombia y Amaya-Perilla \& Palacino-Rodríguez (2012) publican una lista de los odonatos del departamento del Meta.

En años recientes, diferentes autores ampliaron el registro y la distribución de las familias y géneros conocidos para Colombia y describieron nuevas especies asociadas con sus correspondientes estados adultos (Pérez-Gutiérrez, 2003, 2007; Pérez et al. 2007; Bermúdez \& López, 2009; Montes-Fontalvo \& Pérez-Gutiérrez, 2011a, b, AmayaVallejo \& Novelo-Gutiérrez, 2011 y Rojas-Riaño, 2011). En Colombia, igual que para Sur América, la mayor riqueza de especies está en las familias Libellulidae (Anisoptera) y Coenagrionadae (Zigoptera). Sin embargo, el conocimiento sobre su distribución, los requerimientos del hábitat y la ecología están poco documentados (von Ellenrieder \& Garrison, 2009).
OrdenPlecoptera. Los trabajos realizados durante la última década han contribuido a incrementar el conocimiento de la diversidad y la distribución del orden en el país. Plecoptera está representado por la familia Gripopterygidae y el género Claudioperla, es registrada para los Andes del sur de Nariño (Barreto et al. 2005, Zúñiga et al. 2009) y Perlidae, con los géneros Klapalekia (conocido en los Andes orientales de la Sabana de Bogotá), Anacroneuria dominante en Colombia y el Neotrópico y Macrogynoplax, registrado únicamente en tierras bajas de la región amazónica (Zúñiga \& Stark, 2007; Zúñiga et al. 2001, 2007). El registro actual de Anacroneuria muestra una amplia distribución altitudinal (50-3600 msnm), la mayoría de ellas citadas para la región natural andina y pacífica y en menor proporción para la caribe y la amazónica.

Varios autores contribuyeron en los últimos años al avance del conocimiento del orden, mediante la descripción de nuevas especies y el registro y ampliación del ámbito de distribución para los géneros y las especies, particularmente de la familia Perlidae (Rojas \& Baena, 1993; Zamora \& Rossler, 1995, 1997; Stark et al. 1999, 2002, 2009; Stark y Zúñiga, 2003; Zúñiga \& Stark, 2002, 2007; Zúñiga et al. 2007, 2009; Zúñiga, 2010 y Bohórquez et al. 2011). Claves taxonómicas para la identificación de los estados inmaduros y los individuos adultos del orden en Sur América y Colombia se encuentran en Stark et al. (1999, 2009) y Froehlich (2009).

Orden Hemiptera. Son los insectos conocidos popularmente como chinches de agua. Se distinguen, los semiacuáticos (infraorden Gerromorpha) que viven en la película superficial del agua y los verdaderos heteróptera acuáticos (infraorden Nepomorpha), bajo la superficie del agua. Los registros pioneros del grupo fueron hechos por Roback \& Nieser (1974) en los llanos orientales y Álvarez \& Roldán (1983) en Antioquia. Las familias de mayor frecuencia y riqueza específica son Naucoridae y Veliidae. Entre los Heteroptera, los Gerromorpha constituyen el grupo mejor conocido en el país. Aristizábal (2002) estudió los taxones de este infraorden en la película superficial del agua. Molano et al. (2005) reportan un listado de las especies de Gerromorpha, con información sobre su distribución geográfica y altitudinal. Aportes importantes al conocimiento de los heterópteros en Colombia y la descripción de nuevas especies fueron hechas por (Polhemus \& Manzano, 1992; Polhemus \& Polhemus, 1995; Padilla \& Nieser, 2003; Padilla, 2010a,b, 2012a,b; Molano \& Camacho, 2006; Morales-Castaño \& Molano-Rendón, 2008; Posso \& González, 2008; Mazzucconi et al. 2009 y Sites \& Álvarez, 2010). Es de destacar la producividad científica de Dora P. Padilla en los últimos años (Padilla, 2013, 2014, 2015 y 2016; Parra-Trujillo et al. 2014). Esta información está relacionada especialmente con los departamentos de Antioquia, Risaralda, Quindío, Nariño, Valle del Cauca, centro y suroccidente del país. 
Orden Coleoptera. Es un grupo megadiverso en la zona Neotropical, con representantes acuáticos o semiacuáticos en la vegetación litoral. Los primeros trabajos fueron hechos por Wooldrige (1973, 1976), con énfasis en la descripción de nuevas especies y registros de los géneros en el norte del país, cuenca del Río Cauca y Magdalena y el Urabá antioqueño. Las familias de mayor abundancia y riqueza son Elmidae, Ptilodactylidae y Psephenidae y, en general, están asociadas a aguas de buena calidad ambiental (Roldán, 2003; Arias-Díaz et al. 2007; Zúñiga \& Cardona 2009). Otros autores contribuyeron con el conocimiento del grupo en la región Neotropical, afín con la fauna de coleópteros del país (Spangler, 1981 y Spangler \& Santiago, 1987, 1992). Posteriormente, Machado (1988), Roldán (1988), Ramos (1997), Manzo (2005, 2006), Caupaz-Flórez et al. (2006), Arias Díaz et al. (2007) y Gutiérrez et al. (2009), aportaron información a la riqueza del orden, especialmente en los departamentos de Antioquia, Tolima, Valle del Cauca, suroccidente y centro del país. Elmidae fue la familia de mayor diversidad y ámbito de altura, con predominio de los géneros Heterelmis, Macrelmis, Cylloepus, Microcylloepus, y Disersus.

Orden Megaloptera. La familia Corydalidae y el género Corydalus es la registrada con mayor frecuencia (Roldán, 2003; Zúñiga \& Cardona, 2009). Contreras-Ramos (1998) contribuyó al conocimiento del orden en Colombia con nuevos registros de distribución y la descripción de nuevas especies de Corydalus presentes en el país. ContrerasRamos (2009) elaboró una clave taxonómica ilustrada para larvas y adultos para este orden en Sur América y una sinópsis de las familias Croydalidae y Sialidae, información válida para los taxones que se encuentran en Colombia.

Orden Trichoptera. Los primeros estudios fueron realizados por Correa et al. (1981). Hydropsychidae es la familia de mayor distribución y diversidad en Colombia, seguida de Leptoceridae e Hydroptilidae (Reinoso, 1999; Muñoz, 2004; Guevara et al. 2005, 2007a, b; Reinoso et al. 2007, 2008; Vásquez et al. 2010; Vásquez \& Reinoso 2012 y Vásquez et al. 2013, 2014). En las últimas décadas varios autores contribuyeron al registro y la descripción de nuevos géneros y especies que existen en Colombia con énfasis en la región natural andina, particularmente de las familias Hydropsychidae, Leptoceridae e Hydroptilidae (Flint, 1978; Flint \& Wallace, 1980; Holzenthal, 1988a, b; Holzenthal \& Flint, 1995; Holzenthal \& Blahnik, 1995; Blanik, 1998 y Muñoz-Quesada, 1997). Flint (1991) publica un estudio sobre los tricópteros de Antioquia y describe una nueva especie Mortoniella roldani (en reconocimiento al autor de este artículo). Claves taxonómicas para la identificación de las formas inmaduras y los individuos adultos de Trichoptera presentes en Colombia, solo están documentadas regionalmente en Antioquia (Roldán, 1988; Flint, 1991 y Posada \& Roldán, 2003). La información consignada por Angrisano \& Sganga
(2009) a nivel sudamericano y neotropical y Springer et al. (2010) en Costa Rica, incluyen varios taxones que se encuentran en Colombia.

Orden Lepidoptera. Es un grupo aun poco estudiado; los primeros reportes fueron hechos por Berg (1871) sobre la familia Piralidae. Dyar (1914) reporta los lepidópteros de la zona del Canal de Panamá. Romero \& Navarro (2009) aportaron información sobre las familias más comunes con representantes acuáticos o semiacuátios y elaboraron una clave taxonómica ilustrada para larvas y adultos de estos taxones en Suramérica, varios de ellos presentes en Colombia.

Orden Diptera. Es un grupo megadiverso; Welkenson (1979) reportó los Tabanidae de los departamentos del Chocó, Valle del Cauca. Bedoya \& Roldán (1984) realizaron evaluaciones sobre la riqueza y la distribución altitudinal de los estados larvarios de las familias del orden en el departamento de Antioquia. Wayne \& Hogue (1986) reportan una nueva especie de Ephydridae Diedrops roldanorum, Hogue \& Bedoya (1989) describen una nueva especie de Blephariceridae en Antioquia Paltostima roldani, y Hogue (1990) reporta una nueva especie de Blephariceridae Maruina roldanorum; (estas tres especies fueron nominadas en reconocimiento al autor de este artículo). Lizarralde de Grosso (2009) reportó las principales familias de dípteros acuáticos conocidos para Sudamérica, varias de ellas frecuentes en cuerpos de agua del país. Información sobre los trabajos con las familias Simuliidae y Ceratopogonidae, con representantes en el medio acuático, se encuentran en la serie Aquatic Biodiversity in Latin América-ABLA (Coscarón \& Coscarón-Arias, 2007 y Borkent \& Spinelli, 2007).

La familia Chironomidae constituye parte importante de la biomasa de los ambientes lóticos y lénticos y tiene un papel determinante en los ciclos tróficos y el procesamiento de los detritus (Ospina et al. 1999; Paggi, 1999). Abril \& Parra (2007) y Posada-García et al. (2008) generaron información sobre la familia con base en el análisis de los huevos, las larvas, las pupas y los individuos adultos de los sistemas lénticos y lóticos del Páramo de Frontino (3500-4000 msnm). Con base en información de la sabana de Bogotá, Ospina et al. (1999) y Ruiz et al. (2000a, b) elaboraron guías taxonómicas ilustradas para la identificación de genéros de Chironominae, Tanypodinae, Podonominae y Diamesinae. Wiedembrug \& Ospina (2005) publican una clave para la identificación de exhuvias de Tanytarsini (Chironmidae) neotropicales.

\section{Phylum Arthopoda}

\section{Clase Arachnoidea}

Aún no hay un estudio sistemático de este taxón para Colombia. Algunos registros pioneros de los ácaros acuáticos del país fueron hechos por Walter (1912), Lundblad (1953) 
y Viets (1956). Recientemente, Rosso de Ferradás \& Fernández (1995, 2005, 2009) publicaron un listado de las especies con datos biogeográficos y claves taxonómicas ilustradas para las principales familias y géneros de los ácaros acuáticos (Hydrachnidia) de Sur América.

\section{Clase Crustacea}

A esta clase pertenecen los cangrejos de agua dulce. Este grupo de macroinvertebrados, en especial del orden Decapoda es el mejor conocido. Rodríguez (1972), describe Brachyura, Trichodactylidae de Venezuela y Colombia. Rodríguez (1981) distingue dos familias Trichodactylidae de zonas geográficas bajas y Pseudothelpusidae de regiones de montaña y Rodríguez (1985) describe los Pseudothelphusidae de Colombia. Von Prahl (1988) reporta los cangrejos de agua dulce del departamento de Antioquia. Rocha (1994) realiza una extensa revisión de la Familia Pseudothelpusidae, género Neostrengeria en la cordillera oriental de Colombia. Campos (2003), hace la revisión del género Hypolobocera (Pseudothelphusidae); Watling, (2003), reporta una nueva especie de Hyalella para Colombia; Valencia \& Campos (2007) evalúan el género Macrobrachium (Palaemonidae); Campos \& Guerra (2008), establecen la propuesta de sinonimia para las especies de cangrejo dulceacuícola Hypolobocera solimanie - Hypolobocera triangula de Colombia, y Arteta-Bonivento (2009) hace un estudio sobre los cangrejos en el delta del Río Rancheria, Colombia.

\section{Phylum Mollusca}

Los primeros registros los realizó Fuhrmann \& Mayor (1914) a partir de su viaje de exploración científica en Colombia. Posteriormente, Patiño-González (Hermano Daniel) (1941), Pilsbry (1955), Prain (1956), Malek \& Little (1971), aportaron al conocimiento del grupo, mediante la descripción de nuevas especies en diferentes familias. En años recientes, Soler (1983) y Gómez \& Velásquez (1999), contribuyeron al estudio taxonómico y ecológico de los caracoles pulmonados de agua dulce en la sabana de Bogotá y los moluscos de la reserva ecológica del Cerro San Miguel en Medellín.

\section{Estudios ecológicos}

Los trabajos pioneros sobre la ecología acuática en Colombia se iniciaron en los años setenta con los estudios sobre la contaminación del río Medellín y otras corrientes de agua en el departamento de Antioquia (Roldán et al. (1973); Pérez\& Roldán, (1978); Matthias \& Moreno, (1983). Posteriormente Quintero \& Rojas (1987), Rojas et al. (1995); Rojas \& Zúñiga (1996); Zamora (1996, 2002); Zúñiga et al. (1997), Ballesteros et al. (1997), Ramos (1997), Roldán (2001), Urrutia (2005), Gutiérrez et al. (2009), García et al. (2009), Zúñiga (2010) y Giraldo (2012), registraron en el Valle del Cauca y la región suroccidental la fauna de Ephemeroptera, Trichoptera,
Odonata y Coleoptera, su distribución altitudinal, los aspectos ecológicosy la relación con la calidad del agua y el hábitat. Chará et al. (2009) y Giraldo (2012) evaluaron la composición de la comunidad de los macroinvertebrados bentónicos en las corrientes de bajo orden en el sur y centro del país.

Zamora (1995, 1996, 2002, 2010) y Serna \& Zamora (2004) analizaron la similitud de la comunidad de macroinvertebrados bentónicos entre los ecosistemas lénticos ubicados en la costa pacífica caucana y el piedemonte amazónico, así como también los diferentes aspectos ecológicos y biogeográficos de las corrientes hídricas del departamento del Cauca. Guevara-Cardona et al. (2005), Arias et al. (2007), Guevara-Cardona et al. (2007a, b), Vásquez-Ramos \& Reinoso-Flórez (2012), reportaron la estructura y distribución de coleópteros y tricópteros y su relación con la calidad del agua en varias corrientes del departamentos del Tolima. Casas et al. (2006) analizaron la composición y la distribución de los efemeropteros en algunos ríos del Chocó. Rincón $(1996,1999)$ y Romero et al. (2006) describen la distribución espacial y altitudinal de los tricópteros en los Andes orientales y Rincón \& Castro (2008) analizan el efecto del caudal sobre los patrones de emergencia de los individuos de este orden.

La estructura de la comunidad de Trichoptera y su dinámica espacio-temporal en un gradiente altitudinal de la cuenca del río Manzanares fue estudiada por Serna (2003); RúaGarcía (2012) reportó la composición y distribución de los órdenes Ephemeroptera, Plecoptera y Trichoptera en cuatro ríos de la Sierra Nevada de Santa Marta. Pérez \& Gutiérrez (2003) realizan el estudio biotaxonómico de los odonatos del distrito de Santa Marta, Rodríguez et al. (2011) evaluaron los grupos funcionales alimentarios de macroinvertebrados acuáticos en el río Gaira y Aguirre-Pabón et al. (2012) estudiaron la deriva de macroinvertebrados acuáticos en el río Gaira. Ballesteros (2004), Tamaris-Turizo et al. (2007), Tamaris-Turizo \& Sierra-Labastidas (2009), Zúñiga (2010) y Bohórquez et al. (2011), aportaron al conocimiento del microhábitat, los hábitos alimentarios, los patrones de emergencia y la producción secundaria para el orden Plecoptera en la zona andina del suroccidente y el caribe colombiano. Rincón (2002) analizó las preferencias de diferentes taxones de insectos acuáticos por los microhábitats disponibles en una pequeña corriente altoandina de Boyacá. Realpe (2009), determinó la diversidad del genero Ischnura (Odonata: Coenagronidae) y su relación con la altitud y orogenia de la Cordillera Oriental.

Zamora et al. (1996) evaluaron la composición de las comunidades de los macroinvertebrados acuáticos en pequeñas corrientes en la isla de Gorgona. Longo et al. (2004, 2005, 2009, 2010) analizaron la composición, la riqueza, la densidad, la diversidad y la equidad de la comunidad de macroinvertebrados y el ensamblaje de insectos acuáticos en la Isla Gorgona y su relación con las variables físicas, 
químicas e hidráulicas. Longo et al. (2009) y Longo \& Blanco (2009), analizaron las variables ambientales, físicas y químicas que influyeron en la composición y la estructura del ensamble de la entomofauna acuática en dicha isla.

Chará-Serna et al. (2010, 2012), evaluaron mediante análisis de contenido estomacal la dieta de la entomofauna asociada a los paquetes de hojarasca en corrientes de bajo orden en las zonas protegidas de la ecorregión cafetera de Colombia y definieron el papel trófico de esta fauna. RodríguezBarrios et al. (2011) determinaron la riqueza, la abundancia y la biomasa de los grupos funcionales alimentarios de la comunidad de macroinvertebrados y su variación espaciotemporal en una corriente de la Sierra Nevada de Santa Marta. Boyero et al. (2011a, b), analizaron los patrones de la distribución a nivel global de los macroinvertebrados detritívoros y de la diversidad latitudinal, así como sus implicaciones en la pérdida de la biodiversidad en las corrientes ubicadas en zonas de clima variable.

Nazarova et al. (2004) realizaron observaciones sobre las deformidades del aparato bucal de las larvas de Chironomidae en los canales laterales de la Ciénaga Grande de Santa Marta, en una comunidad dominada por Goeldichironomus y Chironomus. Adicionalmente, Monsalve (2004) y Abril \& Parra (2007) utilizaron los fósiles de esta familia para valoraciones sedimentarias del páramo de Frontino en Antioquia y discutieron su utilización como indicadores del cambio climático.

Rodríguez-Barrios et al. (2007) estimaron la variación en la densidad de la deriva de los macroinvertebrados acuáticos y su aporte de materia orgánica en términos de la biomasa en una pequeña corriente tropical de montaña en los cerros orientales de Bogotá. Tamaris-Turizo (2009) evaluó el transporte de la materia orgánica y la deriva de los macroinvertebrados acuáticos en un río en la región Caribe. Quiñonez et al. (1998) analizaron estos tópicos en la zona de ritral del río Medellín. Carmona et al. (2010) evalúan la calidad de las aguas en una vereda El Colorado del municipio de Guarne (Ant), Chará et al. (2007, 2008, 2011) evaluaron la transformación del paisaje hacia monocultivos agrícolas y ganaderos en las microcuencas asociadas al río La Vieja (Valle del Cauca). Los resultados de estos trabajos mostraron problemas de degradación del suelo, la pérdida de la diversidad biológica y la disminución de la calidad y cantidad de agua, especialmente en corrientes pequeñas. Contreras et al. (2008) evalúan la calidad del agua en cuatro corrientes en Norte de Santander. Longo et al. (2005) discuten aspectos relacionados con la autoecología de dos especies de Lymnaea. Giraldo et al. (2014) determinaron los cambios en el ambiente acuático asociados a la restauración del corredor ribereño en las corrientes de bajo orden afectadas por la ganadería y su impacto sobre las comunidades bentónicas y las características abióticas. Donato-Rondón et al. (2010) muestran los efectos de la eutrofización en la interaccion entre las algas y los herbívoros en una corriente andina.
Castro-Rebolledo et al. (2015) discuten los patrones de emergencia de los insectos acuáticos.

\section{Empleo de los macroinvertebrados acuáticos como indicadores de calidad del agua}

Las siguientes son las razones por las cuales se consideran los macroinvertebrados como los mejores indicadores de calidad del agua: son abundantes, de amplia distribución y fáciles de recolectar; son sedentarios en su mayoría $\mathrm{y}$, por tanto, reflejan las condiciones de su habitat; son relativamente fáciles de identificar; representan los efectos de las variaciones ambientales de corto tiempo; proporcionan información para integrar efectos acumulativos; poseen ciclos de vida largos (semanas y/o meses); se reconocen a simple vista; pueden cultivarse en el laboratorio; responden rápidamente a los tensores ambientales y varían poco genéticamente (Roldán, 1999, 2003). Las comunidades de macroinvertebrados presentan diferentes respuestas a la contaminación. Metcalf (1989), distingue tres enfoques principales para evaluar la respuesta de las comunidades de macroinvertebrados a la contaminación. Estos son: el sapróbico, la diversidad y el biótico.

El enfoque sapróbico. El término saprobio, para referirse a la capacidad que tenían ciertos organismos de vivir en determinados niveles de contaminación, fue designado en Alemania por Kolkwitz \& Marsson (1908, 1909). Definieron tres niveles de saprobiedad: a) zona polisapróbica, predominantemente de procesos reductivos, b) zona mesosapróbica, parcialmente reductiva con procesos predominantemente oxidativos y c) zona oligosapróbica, exclusivamente procesos oxidativos. En el sistema saprobio se utilizan todos los organismos acuáticos como indicadores de calidad del agua. También se tienen en cuenta algunos aspectos fisicoquímicos como $\mathrm{DBO}_{5}$, el $\mathrm{NH}_{4}-\mathrm{N}$ y el oxigeno disuelto. La ventaja del sistema saprobio es que incluye gran variedad de taxones y es aplicable a todo tipo de ríos. La desventaja, es que los bioindicadores deben emplearse a nivel de especie y en el neotrópico esta información aun no esta disponible. Al igual que otros sistemas de bioindicación, el sistema sapróbico se desarrolló para dar un valor numérico, conocido como índice sapróbico, el cual va de 1.0 a 4.0 y se obtiene mediante la siguiente ecuación:

$$
\mathrm{S}=\frac{\sum_{1}^{n}(\mathrm{nSi})(\mathrm{Ai})(\mathrm{Gi})}{\sum_{1}^{n}(\mathrm{Ai})(\mathrm{Gi})}
$$

Donde:

S = índice sapróbico

$n$ = número de taxones

$i=$ número de orden de los taxones

$\mathrm{Si}=$ valor de saprobiedad de los taxones

$G=$ peso indicativo de los taxones

A: abundancia de taxones 
El enfoque de la diversidad. Incluye tres componentes fundamentales de las comunidades naturales: riqueza, uniformidad o equidad y un indicativo de abundancia (en el caso de macroinvertebrados, el mas usado es de la densidad, pero bien podría ser biomasa, para describir la respuesta de la comunidad a la calidad ambiental. Una comunidad natural se caracteriza por presentar una alta diversidad de taxones y un número reducido de individuos por taxon; o un bajo número de taxones y muchos individuos por taxon. Esta situación se observa en la naturaleza en lugares como en las profundidades de los lagos y el mar, grandes alturas en las montañas y en sistemas sometidos a temperatures extremas. La contaminación del agua provoca una situación similar, haciendo que ciertas comunidades muy sensibles desaparezcan y otras mas resistentes aumenten en número. Se han desarrollado varios índices para medir la calidad del agua; uno de los más conocidos es el de Shannon-Weaver (1949) expres ada como:

$$
\mathrm{H}^{\prime}=-\sum_{1}^{s}\left(\sum p i\right)(\operatorname{Ln} p i)
$$

Donde:

H’= índice de diversidad de Shannon \& Weaver

$\mathrm{S}=$ número de taxones

$i=$ número de orden de los taxones

$p i$ = indicador de abundancia relativa ( densidad relativa o biomasa relativa son los más comunes)

$n i=$ número de individuos por taxón en la muestra.

$n$ = número total de individuos en la muestra .

$L n=$ logaritmo natural.

El enfoque biótico. Incluye los aspectos esenciales de la saprobiedad, combinando una medida cuantitativa de diversidad de especies con la información cualitativa sobre la sensibilidad ecológica de taxones de individuos en una expresión numérica simple. Beck (1955) propuso el índice biótico en los Estados Unidos basado en la relación entre especies intolerantes y tolerantes a la contaminación; los valores se encuentran entre 0 y 10. Este índice se expresa como:

$$
\mathrm{I}=2 \mathrm{Si}-2 \mathrm{~S} 1
$$

Donde:

$\mathrm{Si}=$ número de especies intolerantes

S1 = número de especies tolerantes

Cualquier índice tiene su utilidad y valor de acuerdo con el criterio con que se use y con el conocimiento que se tenga de la fauna local.

\section{El método BMWP para Colombia (BMWP/Col)}

El Biological Monitoring Working Party (BMWP) fue establecido en Inglaterra en 1970, como un método simple y rápido para evaluar la calidad del agua usando los macroinvertebrados como bioindicadores. Las razones para ello fueron básicamente económicas y por el tiempo que se necesita invertir. El método sólo requiere llegar hasta nivel de familia y los datos son cualitativos (presencia o ausencia). El puntaje va de 1 a 10 de acuerdo con la tolerencia de los diferentes grupos a la contaminación orgánica. Las familias más sensibles como Perlidae y Oligoneuriidae reciben un puntaje de 10; en cambio, las más tolerantes a la contaminación, por ejemplo, Tubificidae, reciben una puntuación de 1.0 (Armitage et al. 1983). La suma de los puntajes de todas las familias proporciona el puntaje total BMWP.

La bioindicación en Colombia se remonta a los años setenta con los trabajos en el río Medellín (Roldán et al. 1973). Posteriormente Matthias \& Moreno (1983) realizaron un estudio fisicoquímico y biológico del mismo río, utilizando los macroinvertebrados como indicadores de la calidad del agua. Bohórquez \& Acuña (1984) realizaron los primeros estudios para la sabana de Bogotá. (Zúñiga et al. (1993) hicieron una adaptación de este método para algunas cuencas del Valle del Cauca. Reinoso (1999) realizó un estudio del río Combeima en el Departamento del Tolima. Zamora (2000) hizo una adaptación del índice BMWP para la evaluación de la calidad de las aguas epicontinentales en Colombia. Roldán (2001) aplica esta metodologia para la cuenca de Piedras Blancas en el Departamento de Antioquia. Riss et al. (2002) establecen valores de biondicación para la Sabana de Bogotá. Roldán (2003) adapta el sistema del BMWP para evaluar la calidad del agua en Colombia mediante el uso de los macroinvertebrados acuáticos. Con base en el conocimiento que actualmente se tiene en Colombia sobre los diferentes grupos de macroinvertebrados hasta el nivel de familia, propone utilizar el método BMWP/Col, como una primera aproximación para evaluar los ecosistemas acuáticos de montaña. La Tabla 2 presenta las familias y su valoración de acuerdo a su grado de adaptación a las diferentes calidades de agua. Cada región, tanto en Colombia como en Latinoamérica, han hecho sus propias valoraciones de puntajes de acuerdo a las sus experiencias (Zamora \& Sarria, 2001; Sánchez-Herrera, 2005; Zúñiga 2009, Springer et al.2010).

Las formas inmaduras de la entomofauna tienen un buen potencial como bioindicadoras, además de ser una comunidad diversa, abundante y de amplia distribución altitudinal en los ecosistemas hídricos de Colombia. El género Anacroneuria (Plecoptera: Perlidae), es considerado uno de los grupos de mayor sensibilidad a la degradación del hábitat y del enriquecimiento de la carga orgánica residual (Roldán, 2003; Zúñiga, 2010). Entre los géneros de Ephemeroptera más sensibles se encuentran Lachlania (Ologineuriidae), Haplohyphes (Leptohyphidae), Mayobaetis, Andesiops (Baetidae) Atopophlebia y Thraulodes (Leptobhlebiidae); mientras que Camelobaetidius, Baetodes (Baetidae), Leptohyphes y Tricorythodes (Leptohyphidae) son de amplio espectro ambiental (Roldán, 2003; Zamora, 
Tabla 2. Puntajes de las familias de macroinvertebrados acuáticos para el índice BMWP/Col (Roldán, 2003).

\begin{tabular}{|c|c|}
\hline Familias & Puntajes \\
\hline $\begin{array}{l}\text { Anomalopsychidae, Atriplectididae, Blepharoceridae, Calamoceratidae, Ptilodactylidae, Chordodidae, Gomphidae, Hidridae, Lampyridae, } \\
\text { Lymnessiidae, Odontoceridae, Oligoneuriidae, Perlidae, Polythoridae, Psephenidae }\end{array}$ & 10 \\
\hline $\begin{array}{l}\text { Ampullariidae, Dytiscidae, Ephemeridae, Euthyplociidae, Gyrinidae, Hydrobiosidae, Leptophlebiidae, Philopotamidae, } \\
\text { Polycentropodidae, Xiphocentronidae. }\end{array}$ & 9 \\
\hline $\begin{array}{l}\text { Gerridae, Hebridae, Helicopsychidae, Hydrobiidae, Leptoceridae, Lestidae, Palaemonidae, Pleidae, Pseudothelpusidae, Saldidae, } \\
\text { Simuliidae, Veliidae. }\end{array}$ & 8 \\
\hline $\begin{array}{l}\text { Baetidae, Caenidae, Calopterygidae, Coenagrionidae, Corixidae, Dixidae, Dryopidae, Glossossomatidae, Hyalellidae, Hydroptilidae, } \\
\text { Hydropsychidae, Leptohyphidae, Naucoridae, Notonectidae, Planariidae, Psychodidae, Scirtidae. }\end{array}$ & 7 \\
\hline Aeshnidae, Ancylidae, Corydalidae, Elmidae, Libellulidae, Limnichidae, Lutrochidae, Megapodagrionidae, Sialidae, Staphylinidae. & 6 \\
\hline Belostomatidae, Gelastocoridae, Hydropsychidae, Mesoveliidae, Nepidae, Planorbiidae, Pyralidae, Tabanidae, Thiaridae & 5 \\
\hline Chrysomelidae, Stratiomyidae, Haliplidae, Empididae, Dolicopodidae, Sphaeridae, Lymnaeidae, Hydraenidae, Hydrometridae, Noteridae. & 4 \\
\hline Ceratopogonidae, Glossiphoniidae, Cyclobdellidae, Hydrophilidae, Physidae, Tipulidae. & 3 \\
\hline Culicidae, Chironomidae, Muscidae, Sciomyzidae, & 2 \\
\hline Tubificidae & 1 \\
\hline
\end{tabular}

1996; Zúñiga et al. 1997, Zúñiga \& Cardona, 2009). Los géneros más sensibles del orden Trichoptera son Triplectides (Leptoceridae), Rhyacopsyche (Hydroptiliidae), Chimara (Philopotamidae), Marilia (Odontoceridae) y Phylloicus (Calamoceratidae). Los géneros Leptonema (Hydropsychidae) y Atanatolica (Leptoceridae) tienen un ámbito amplio, con adaptaciones a los ecosistemas con degradación incipiente (Roldán, 2003; Zúñiga et al.1993; Zamora, 1996; Ballesteros et al. 1997; Guevara et al. 2007a, b; Zúñiga \& Cardona 2009); Forero et al. (2013); Forero \& Reinoso, 2013); Vásquez et al. (2013, 2014).

\section{Estandarización de protocolos de trabajo}

En Colombia no hay protocolos normalizados para los trabajos en la bioindicación de la calidad del agua, aplicados a nivel regional o nacional, que faciliten las comparaciones entre los estudios realizados en las diferentes zonas del país. Es necesario trabajar en la estandarización de estos protocolos de muestreo, con énfasis en los grandes ríos y los cuerpos de agua lénticos, como los humedales o los grandes lagos. Esta homogeneización de protocolos de trabajo de campo, el recuento de los organismos, el análisis de laboratorio y el uso de los índices, son imprescindibles para conseguir los resultados y bases de datos consistentes y comparables (Roldán et al. 2014). Rueda-Delgado (2002) publicó el Manual de Métodos en Limnología, en donde compiló la información general sobre los diferentes métodos de estudio para la biota de mayor importancia en los ambientes acuáticos. Actualmente el Instituto de Hidrología, Meteorología y Estudios Ambientales de Colombia-IDEAM está desarrollando una metodología de evaluación de la calidad del agua para Colombia mediante la utilización de los macroinvertebrados acuáticos como bioindicadores (Sanabria, 2015, (Comunicación personal).
Uno de los problemas en estudios de bioindicación de la calidad del agua en Colombia es el uso indiscriminado de los índices bióticos, tanto en ecosistemas de aguas lóticas como lénticas. Estos ecosistemas tienen características hidrológicas y ecológicas diferentes y una biota adaptada a las condiciones particulares de cada uno de estos ambientes. El índice BMWP es muy popular y, aunque existen algunas adaptaciones de este parámetro biológico a nivel regional, para la validez de su aplicación es importante tener en cuenta la clase de los cuerpos de agua donde se aplica. Este índice ha sido estandarizado para la evaluación de la calidad del agua en corrientes hídricas de bajo y mediano orden, principalmente de la zona andina (Zúñiga et al. 1993; Zamora, 2000, Roldán, 2003).

\section{Estudios taxonómicos detallados}

La implementación y la eficacia de los programas encaminados a la evaluación de la calidad del agua a partir de orgaminasmos bioindicadores, depende en gran medida del soporte sólido de la taxonomía. Este aspecto es fundamental para garantizar la identificación correcta de los diferentes grupos que pueden suministrar la información ecológica, acerca de su respuesta con la calidad ambiental del recurso hídrico. En tal sentido, es importante aunar esfuerzos entre los diferentes grupos que estudian el tema, con el fin de elaborar una guía taxonómica adaptada a la fauna regional típica de las corrientes hídricas, con diferente nivel de deterioro ambiental.

La información sobre la taxonomía y la ecología de algunos de los grupos entomológicos de mayor diversidad, abundancia y biomasa es aún deficiente. Esta situación se presenta para los órdenes Diptera Odonata y Hemiptera, taxones que podrían suministrar información valiosa desde el 
punto de vista de la bioindicación, especialmente para los ambientes lénticos en donde su abundancia y diversidad, en general, es mayor que la que se presenta en los ecosistemas de las aguas corrientes. Por su parte, los moluscos y los anélidos, cuya abundancia y biomasa es muy alta en ambientes contaminados y enriquecidos con materia orgánica, carecen de una información taxonómica más detallada, lleva a generalizaciones e interpretaciones erradas acerca de su potencial como biondicadores de la calidad del agua.

\section{Conclusiones}

La información disponible en Colombia sobre la taxonomía, la ecología y la bioindicación de la calidad del agua no es similar en todos los grupos. Ephemeroptera, Plecoptera y Trichoptera cuentan con mayor información que los demás taxones. Es importante además, profundizar en el estudio de los anélidos, los moluscos y los dípteros, especialmente de la familia Chironomidae.

Es necesario avanzar en la asociación de los estados inmaduros y sus correspondientes formas aladas, así como entre machos y hembras, con el fin de lograr una determinación taxonómica rigurosa.

Es importante definir una metodología unificada para el estudio de los ecosistemas lénticos y los grandes ríos.

La aplicación de la bioindicacion de la calidad del agua se inició en Colombia en la década de los años setenta y ahora está ampliamente extendida en todos los países latinoameicanos.

El gobierno nacional y, en especial con el Ministerio del Medio Ambiente y el Instituto de Hidrología, Meteorología y Estudios Ambientales- IDEAM, deben implementar normas que promuevan la utilización de los macroinvertebrados como bioindicadores en programas de evaluación ambiental de cuerpos de agua. Esta herramienta es un buen complemento a la información de tipo fisicoquímica y bacteriológica de amplia utilización en Colombia y permitirá un análisis integral del recurso hídrico.

\section{Conflicto de intereses}

El autor declara no tener conflicto de intereses.

\section{Bibliografía}

Abril, R. G. y Parra, L.N. 2007. Macroinvertebrados acuáticos de páramo de Frontino Antioquia Colombia con énfasis en Chironomidae. Tesis Maestría Universidad Nacional de Colombia, sede Medellín. 60 p.

Aguirre. P, J., Rodríguez, B. J y. Rodulfo, O.T. 2012. Deriva de macroinvertebrados acuáticos en dos sitios con diferente grado de perturbación, Río Gaira, Santa Marta, Colombia. Rev. Intropica. 7 9-19. Santa Marta, Colombia

Alba-Tercedor, J. y Sánchez-Ortega, A. 1988. Un método rápido y simple para evaluar la calidad biológica de las aguas corrientes, basado en el de Hellawel 1978. Limnetica 4: 51-56.
Alba-Tercedor, J.1996. Macroinvertebrados acuáticos y calidad de las aguas de los Ríos. IV Simposio del Agua en Andalucía (SIAGA), Almeria, vol. 2, pp. 203-213.

Altamiranda-Saavedra, M., Pérez-Gutiérrez, L.A. \& GutiérrezMoreno, L.C. 2010. Distribución y respuesta de la comunidad de larvas de odonatos (Insecta: Odonata) a la disponibilidad de sustratos en una ciénaga del Departamento del Atlántico, Colombia. Caldasia 32 (2): 399-410.

Álvarez, L.F. y Roldán, G. 1983. Estudio taxonómico y ecológico de los hemípteros a diferentes pisos altitudinales en el Departamento de Antioquia. Actualidades Biológicas 12 (44): 31-45.

Amaya-Perilla, C \& Palacino-Rodríguez, F. 2012.An update list of the dragonflies (Odonata) of Meta department (Colombia) with forty six new department records.Bulletin of American Odonatology 11 (2): 29-38.

Amaya-Vallejo, V. \& Novelo-Gutiérrez, R. 2011. The larva of Palaemnema mutans Calvert 1931 (Odonata: Platystictidae). Zootaxa 3049: 59-63.

Angrisano, E.B. \& Sganga, J.V. 2009. Trichoptera. Pp. 255-307. En: Domínguez, E. \& Fernández, H. (Eds). Macroivertebrados bentónicos sudamericanos. Sistemática y biología. Fundación Miguel Lillo. Tucumán, Argentina.

Arango, M.C. y Roldán, G. 1983. Estudio de las larvas acuáticas del orden Odonata a diferentes pisos altitudinales en el Departamento de Antioquia. Actual. Biol. 12 (46): 91-104.

Arias-Díaz, D., Reinoso-Flórez, G., Guevara-Cardona, G. \& Villa-Navarro, F.A. 2007. Distribución espacial y temporal de los coleópteros acuáticos en la cuenca del Río Coello (Tolima, Colombia). Caldasia 29 (1): 177-194.

Armitage, P. D., D. Moss y M.T. Furse. 1983, The performance of a new biological water quality score system based on macroinvertebrates over a wide range of umpollutes runnigwaters sites. Water Res, 17: 33-347.

Aristizábal, H. 2002. Los hemípteros de la película superficial del agua en Colombia. Parte I: Familia Gerridae. Academia Colombiana de Ciencias Exactas Físicas y Naturales. Colección Jorge Álvarez Lleras No 20, Bogotá.

Arteta-Bonivento, R. 2009. Cangrejos en el delta del río Ranchería, Riohacha (Colombia) (Crustacea: Decapoda: Brachyura). Bol. Cient. Mus. Nat. 13 (1): 140-152.

Astudillo, M.R. 2005. Variación altitudinal de las náyades de los géneros de Odonata (Insecta) en algunos cuerpos de agua del suroccidente colombiano. Tesis de pregrado. Universidad del Valle. Programa de Biología. Santiago de Cali. 55 p.

Ball, I.R. 1969. An annoted checklist of freshwater Tricladida of the Neartic Neotropical regions.Candian Journal of Zoologia 47: 59-64.

Ball, I.R. 1980. Freshwater planarians from Colombia; a revision of Fuhrmans' types.Bijdragen tot Dierkunde 50: 235-242.

Ballesteros, Y.V. 2004. Contribución al conocimiento del género Anacroneuria (Pecoptera: Perlidae) y su relación con la calidad del agua en el río Riofrío (Valle del Cauca). Tesis de Maestría en Ingeniería Sanitaria y Ambiental. Universidad del Valle. Cali, Colombia. 90p. 
Ballesteros, Y.V., Zúñiga, M. del C. y Rojas, A.M. 1997. Distribution and structure of the order Trichoptera in various drainages of the Cauca River basin, Colombia, and their relationships to water quality. Pp. 19-23. En: Holzenthal, R.W. y Flint, O. Jr. (Eds.). Proceedings of the 8th International Symposium on Trichoptera, 1995.Ohio Biological Survey, Columbus, Ohio, USA.

Barreto, V.G., Reinoso, G., Guevara, G. y Villa, F.A. 2005. Primer registro de Gripopterygidae (Insecta: Plecoptera) para Colombia. Caldasia 27: 243-246.

Beck, W. M. 1955. Suggested method for reporting biota data. Sewage Ind. Wastes, 27: 1193-1197.

Bedoya, I. y Roldán, G. 1984. Estudio de los dípteros acuáticos en diferentes pisos altitudinales en el Departamento de Antioquia. Revista Asociación Colombiana de Ciencias Biológicas 2 (2): 113-134.

Berg, C. 1871. Beitrage zu den Pyralidinen Sudamerikas. Ent. Zeit. Entomol. Verein. Stettin 37: 342-355.

Bermůdez, C. 2005. Clave para los imagos de los géneros de Libellulidae (Odonata:Anisoptera) del Valle del Cauca, Colombia. Boletín del Museo de Entomología de la Universidad del Valle 6 (1): 7-22.

Bermůdez, C. y López-Victoria, M. 2009. Primeros registros de libélulas (Odonata: Anisoptera) en la Isla Malpelo, Colombia. Revista Colombiana de Entomología 35 (2): 286-287.

Birk, S. \& D. Hering. 2006. Direct comparison of assessment methods using benthic macroinvertebrates: a contribution to the EU Water Framework Directive intercalibration exercise. Hydrobiologia 566 (1): 401-415.

Blahnik, R.J. 1998. A revisión on the Neotropical species of the genus Chimarra, subgenus Chimarra (Trichoptera: Philopotamidae). Memoirs of the American Entomological Institute 59: 1-318.

Bohórquez, H., Reinoso, G. y Guevara, G. 2011. Seasonal size distribution of Anacroneuria (Plecoptera: Perlidae) in an Andean tropical river. Revista Colombiana de Entomología 37 (2): 305-312.

Bohórquez, A. y A. Acuña. 1984. Inventario de las morfofamilias de las clases Gasterópoda y Clitelata como bioindicadores limnológicos de la laguna de La Herrera. p. 32. Memorias XIX Congreso Nacional y III Grancolombiano de Ciencias Biológicas, Bucaramanga, UniversidadIndustrial de Santander, $70 \mathrm{p}$.

Borkent, A. y Spinelli, G.R. 2007. Neotropical Ceratopogonidae. En: Adis, J., Arias, J.R., Rueda-Delgado, G. \& Wantzen, K.M. (Eds.). Aquatic Biodiversity in Latin America (ABLA). Vol. 2. Pensoft, Sofia, Moscow. Serie ABLA. 198 p.

Boyero, L., Pearson, R., Dudgeon, G., Graca, M.A.S., Gessner, M.O., Albariño, R.J., Ferreira, V., Yule, C.M., Boulton, A.M., Arunachalam, J., Callisto, M., Chauvet, E., Ramírez, A., Chará, J., Moretti., J.F., Goncalves, J.F. Jr., Helson, J.E., Chará-Serna, A.M., Encalada, A.C., Davies, J.N., Lamothe, S., Cornejo, A., Castela, J., Li, A.O.Y., Buria, L.M., Villanueva, V.D., Zúñiga, M.del C. y Pringle, C.M. 2011a. Global distribution of a key trophic guild contrasts with common latitudinal diversity patterns. Ecology 92 (9): 1839-1848.

Boyero, L., Pearson, R.G., Dudgeon, D., Ferreira, V., Graca, M.A.S., Gessner, M.O., Boulton, A.J., Chaubet, E., Yule, C.M., Albariño, R.J., Ramírez, A., Helson, J.E., Callisto, M., Arunachalam, M., Chará, J., Figueroa, R., Mathooko, J.M., Moretti, M.S., Goncalves, J.F. Jr., Chará-Serna, A.M., Davies, J.N., Encalada, E.A., Lamothe, S., Buria, L.M., Castela, J., Cornejo, A., Li, A.O.Y., Erimba, C.M., Villanueva, V.D., Zúñiga, M. del C., Swan, C.M. y Barmuta, L.A. 2011b. Global patterns of stream detritivore distribution: implications for biodiversity loss in changing climates. Global Ecology and Biogeography 21: 134-141.

Brillouin, L. 1951. Maxwells demon cannot operate: information and entropy. I and II. Journ. Appl. Phys., 22: 334-343

Camargo, C. \& Rozo, M.P. 2003. Colombian Darien Ephemeroptera. Pp. 291-292. En: Gaino, E. (Ed.). Research Update on Ephemeroptera \& Plecoptera.University of Perugia, Perugia, Italy.Campos, $\mathrm{M}$ and Rodríguez, G. 1985. A new species of Neostrengeria (Crustacea: Decapoda: Pseudothelphusidae) with notes on geographical distribution of the genus. Proc. Biol. Sci. Wash. 98 (3), 1985, pp. 718-727.

Campos, M. 2003. A review of the freshwater crabs of the genus Hypolobocera Ortmann, 1897 (Crustacea: Decapoda: Brachyura: Pseudothelphusidae), from Colombia Proceedings of the Biological Society of Washington 116 (3):754-802. 2003.

Campos, M \& Guerra, L. 2008. Propuesta de sinonimia para las especies de cangrejo dulceacuícola Hypolobocera solimanie-Hypolobocera triangula de Colombia. Rev. Biol. Trop. vol.56, n.3, pp. 987-994.

Carmona, Y., Herrera, R. y Roldán, G. 2010. Estudio socioeconómico y ambiental de la vereda el Colorado, Municipio de Guarne, Antioquia. Academia Colombiana de Ciencias Exactas Físicas y Naturales. Colección Jorge Álvarez Lleras No. 26. Santafé de Bogotá.

Carsten von der Ohe, P., Pru, R. Bernhard Schafer, M. Liess, E. de Deckere \& W. Brack. 2007. Water quality indices across Europe-a comparison of the good ecological status of five river basins. Journal of Environmental Monitoring 9 (9): 970-978.

Castro-Rebolledo, M. I. \& J. C. Donato-Rondón, 2015. Emergence patterns in tropical insects: The role of water discharge frequency in an Andean Stream. Annales de Limnologie 51 (2): 147-155.

Casas-Córdoba, L., Córdoba-Aragón, K. E., Asprilla-Murillo, S. y Mosquera, Z. 2006. Composición y distribución del Orden Ephemeroptera en los ríos Tutunendo y Catugadó, Quibdó-Chocó (Colombia). Asociación Colombiana de Limnología -Neolimnos 1: 92-97.

Caupaz-Flórez, F., Reinoso, G., Guevara, F.A. \& Villa, F.A. 2006. Diversidad y distribución de la familia Elmidae (Insecta: Coleoptera) en la cuenca del río Prado (Tolima, Colombia). Asociación Colombiana de LimnologíaNeolimnos 1: 106-116. 
Ceballos A. 2004. Contribucion al conocimiento y distribución de los odonatos (Insecta: Odonata) en Colombia. Tesis de pregrado. Universidad Nacional de Colombia.

Chará J., Pedraza, G., Giraldo, L.P e Hincapié, D. 2007. Efecto de corredores ribereños sobre el estado de quebradas en la zona ganadera del río La Vieja, Colombia. Revista Agroforestería de las Américas 45: 72-78.

Chará, J., Pedraza, G. y Giraldo, L.P. 2008. Corredores ribereños como herramienta de protección de ambientes acuáticos en zonas ganaderas. Pp 111-130. En: Murgueitio, E., Cuartas, C. \& Naranjo, J.F. (Eds.). Ganadería del futuro: Investigación para el desarrollo. Fundación CIPAV. Santiago de Cali, Colombia.

Chará, J., Giraldo, L.P., Chará-Serna, A.M. y Pedraza, G.X. 2011. Beneficios de los corredores ribereños de Guadua angustifolia en la protección de ambientes acuáticos en la ecorregión cafetera de Colombia. Efectos sobre la escorrentía y captura de nutrientes. Recursos Naturales y Ambiente 61: 60-66.

Chará, J., Zúñiga, M. del C., Giraldo, L.P., Pedraza, G., Astudillo, A., Ramírez, L. y Posso, C.E. 2009. Diversidad y abundancia de macroinvertebrados acuáticos en quebradas de la cuenca del río La Vieja, Colombia. Pp. 129-142. En: Rodríguez, J.M., Camargo, J.C., Niño, J., Pineda, A.M., Arias, L.M., Echeverry, M.A. \& Miranda, C.L. (Eds.) Valoración de la Biodiversidad en la Ecorregión del Eje Cafetero. CIEBREG. Pereira, Colombia.

Chará-Serna, A.M., Chará, J., Zúñiga, M. del C., Pearson, R.G. y Boyero, L. 2012. Diets of leaf litter associated invertebrates in three tropical streams. Annales de Limnologie - International Journal of Limnology 48: 139-144.

Chará-Serna, A.M., Chará, J., Zúñiga, M. del C., Pedraza, G.X. y Giraldo, L.P. 2010. Clasificación trófica de insectos acuáticos en ocho quebradas protegidas de la ecorregión cafetera colombiana. Universitas Scientiarum 1: 27-36.

Cohn, F. 1853. Über lebende Organismen im Trinkwasser. Z. klin. Med., 4: 229-237.

Contreras-Ramos, A. 1998. Systematics of thedobsonfly genus Corydalus (Megaloptera:Corydalidae). Thomas Say Publications in Entomology: Monographs. Lanham.

Contreras-Ramos, A. 2009. Megaloptera.. En:Domínguez, E. \& Fernández, H. (Eds.). Macroinvertebrados bentónicos sudamericanos: Sistemática y biología, Pp. 233-245. Primera Edición. Fundación Miguel Lillo, San Miguel de Tucumán, Argentina.

Contreras, J., Roldán, G., Arango, A. \& Álvarez, L.F. 2008.Evaluación de la calidad del agua de las microcuencas La Laucha, La Lejía y La Rastrojera utilizando los macroinvertebrados como bioindicadores. Municipio de Durania, departamento Norte de Santander, Colombia. Revista de la Acdemia Colombiana de Ciencias Exactas, Físicas y Naturales 32 (123): 171-193.

Correa, M., Machado, T. y Roldán, G. 1981. Taxonomía y ecología del orden Trichoptera en el Departamento de Antioquia a diferentes pisos altitudinales. Actualidades Biológicas 10 (36): 35-48.
Coscarón. S. \& Coscarón-Arias, C. 2007. Neotropical Simuliidae (Diptera: Insecta). Aquatic Biodiversity of Latin America (ABLA Series).Vol. 3. Pensoft Publishers, Sofia, Moscow.

Cruz, L. 1986. Contribución a los estudios taxonómicos de Odonata Zigoptera de Colombia: descripción de una nueva especie de Cianallagma (Odonata: Coenagrionidae). Caldasia 14 (68/70): 743-747.

De Pauw, N \& H. A. Hawkes. 1993, Biological monitoring of water quality, en: River quality monitoring and control, W. J. Walley y S. Judd, eds., publicado por Aston University, Reino Unido, 249 p.

De Marmels, J. 1982a. Cuatro náyades nuevas de la familia Libellulidae (Odonata: Anisoptera). Boletín de Entomología Venezolana 2 (11): 94-101.

De Marmels, J. 1982b. Dos náyades nuevas de la familia Aeshnidae (Odonata: Anisoptera). Boletín de Entomología Venezolana 2 (12): 102-106.

De Marmels, J. 2001. Aeshna (Hesperaeschna) condor sp. nov. from the Venezuelan Andes, with a redescription of $A$. (H.) joannisi, comments on other species, and description of larvae (Odonata: Aeshnidae). International Journal of Odonatology 4 (2): 119-134.

Dyar, H.G. 1914. Report of Lepidoptera of Smithsonian Biological Survey of Panamá Canal Zone. Proc. US. Nat. Mus. 47: $139-150$

Días, L., Bacca, T., Navarro, L., Acevedo, F., Benavides, P. y Fiuza-Ferreira, P.S. 2011. Association of nymphs and adults of Ephemeroptera (Insecta) using the amplified fragment length polymorphism (AFLP) technique. Annales de Limnologie - International Journal of Limnology 47: 151-157.

Domínguez, E. \& Fernández, H.(Eds.). 2009. Macroinvertebrados bentónicos sudamericanos: Sistemática y biología. Primera Edición. Fundación Miguel Lillo. San Miguel de Tucumán, Argentina.

Domínguez, E. \& Zúñiga, M. del C. 2003. First generic record and description of a new species of Ulmeritoides (Ephemeroptera: Lepophlebiidae) from Colombia. Pp. 4345. En: Gaino, E. (Ed.). Research update on Ephemeroptera and Plecoptera. University of Perugia, Italy.

Domínguez, E. \& Zúñiga, M. del C. 2009. First description of the nymph of Farrodes roundsi (Traver) (Ephemeroptera: Leptophlebiidae, Atalophlebiinae) with comments on its phylogenetic relationships. Aquatic Insects 31 (Suppl. 1): 73-81.

Domínguez, E., Zúñiga, M. del C. y Molineri, C. 2002. Estado actual del conocimiento y distribución del orden Ephemeroptera (Insecta) en la región amazónica. Caldasia 24 (2): 459-469.

Donato-Rondón, J. C., S. J. Morales-Duarte \& M. I. CastroRebolledo. 2010. Effects of eutrophication on the interaction between algae and grazers in an Andean stream. Hydrobiologia 657 (1): 159-166.

Flint, Jr., O.S. 1978. Studies of Neotropical caddisflies. XXII: Hydropsychidae of the Amazon basin (Trichoptera). Amazoniana 6: 373-421. 
Flint, Jr. O.S., Wallace, J.B. 1980. Studies of Neotropical caddisflies, XXV: the immature stages of Blepharopus diaphanous and Leptonema columbianun (Trichoptera: Hydropsychidae). Proceeding of the Biological Society of Washington 93 (1): 178-193.

Flint, Jr. O.S. 1991. Studies of Neotropical Caddisflies, XLV:The Taxonomy, Phenology and Faunistics of the Trichoptera of Antioquia, Colombia. Smithsonian Contributions to Zoology 520: 1-113.

Forero, C. A.M. Gutiérrez, C \& Reinoso, F.G. 2013. Evaluación de la calidad del agua del rio Opia (Tolima-Colombia) a través de la fauna de macroinvertebrados acuáticos y parámetros fisicoquímicos. Rev. Caldasia 35 (2): 371- 387.

Forero, C. A.M \& Reinoso, F. G. 2013. Estudio de la familia Baetidae (Ephemeroptera: Insecta) en una cuenca con influencia de la urbanización y agricultura: río AlvaradoTolima. Rev. Asoc. Col. Cienc. (Col.), 25: 12-21.

Froehlich, C. G. 2009. Plecoptera.. En: Domínguez, E. y Fernández, H. (Eds.). Macroinvertebrados bentónicos sudamericanos: Sistemática y biología. Pp. 145-166. Primera Edición. Fundación Miguel Lillo, San Miguel de Tucumán, Argentina.

Fuhrmann, O. 1914. Turbellariés d'eau douce de Colombie. Mémoires de la Société Neuchâteloise des Sciences Naturelles: 793-804.

Fuhrmann, O. \& Mayor, E. 1914. Voyage d'exploration scientifique en Colombie. Mémoires de la Société Neuchâteloise des Sciences Naturelles: 5 (2): 193-201

Gaufin, A. R. \& Tarzwell, C. M. 1952. Aquatic invertebrates as indicators of stream Pollution. Amer. Publ. Health Rep. 67: No. 1.

García, J. F. Cantera, J. Zúñiga, M. del C \& Montoya, J. 2009. Estructura y diversidad de las comunidades de macroinvertebrados acuáticos en la cuenca baja del río Dagua (Andén pacífico vallecaucano-Colombia). Revista de Ciencias de la Universidad del Valle 13: 27-48.

Garzón, C. \& Realpe, E. 2009. Diversidad de odonata (insecta) en la Reserva Natural Cabildo-Verde (Sabana de TorresSantander, Colombia), una aproximación hacia la conservación. Caldasia vol.31, n.2, pp. 459-470.

Gaviria, E. 1993. Claves para las especies colombianas de las familias Naidae y Tubificidae (Oligochaeta, Annelida). Caldasia 17 (2): 237-248.

Giraldo, L.P. 2012. Diversidad y abundancia de larvas de Trichoptera (Insecta) asociadas a pequeñas quebradas andinas del centro y sur occidente colombiano. Tesis de Maestría. Universidad de Costa Rica. Estudios de Posgrado en Biología. San José, Costa Rica. 125 p.

Giraldo, L.P., Chará, J., Zúñiga, M.del C., Chará-Serna, A.M. \& Pedraza, G. 2014. Impacto del uso del suelo agropecuario sobre macroinvertebrados acuáticos en pequeñas quebradas de la cuenca del río La Vieja (Valle del Cauca, Colombia). Rev. Biol. Trop. (Int. J. Trop. Biol. Vol. 62 (Suppl. 2): 203-219.
Gómez, M.I. \& Velásquez, H.I. 1999. Estudio de los moluscos de agua dulce de la Reserva Ecológica Cerro de San Miguel (Caldas, Antioquia, Colombia). Actualidades Biológicas 21 (71): 151-161.

Guevara, C.G. Reinoso, F.G. \& Villa-Navarro, N.F. 2005. Estudio del orden Trichoptera en su estado larval en la cuenca del Río Coello, Departamento del Tolima. Revista de la Asociación Colombiana de Ciencias Biológicas 17: 59-70.

Guevara-Cardona, G. Reinoso-Flórez, G. \& Villa-Navarro, N.F. 2007a. Caddisfly larvae (Insecta: Trichcotera) of the Coello River Basin in Tolima (Colombia): Spatial and temporal patterns and bioecological aspects. Pp. 113-120. En:Bueno-Soria, J., Barba-Álvarez, R. y Armitage, B. (Eds.). Proceeding of the XIIth International Symposium on Trichoptera.The Caddis Press.

Guevara-Cardona, G. López-Delgado,E.O. Reinoso-Flórez, G. \& Villa-Navarro,N.F. 2007b. Structure and distribution of the Trichoptera fauna in a Colombian Andean river basin (Prado, Tolima) and their relationship to water quality. Pp. 129-134. En: Bueno-Soria, J., Barba-Álvarez, R. y Armitage, B. (Eds.). Proceeding of the XII th International Symposium on Trichoptera. The Caddis Press.

Gutiérrez, C. \& Reinoso, G. 2010. Géneros de ninfas del orden Ephemeroptera (Insecta) del Departamento del Tolima, Colombia: listado preliminar. Biota Colombiana 11 (1 y 2): 23-32.

Gutiérrez, C., Zúñiga, M. del C., Van Bodegom, P.M., Chará, J. \& Giraldo, L.P. 2009. Rove beetles (Coleoptera: Staphylinidae) in Neotropical riverine landscapes: characterizing their distribution. Insect Conservation and Diversity 2: 106-115.

Hincks, W.D. 1934. Odonata (Paraneuroptera) from Perú and Colombia. Entomologist's Record and Journal of Variation 46: 77-81.

Hogue, Ch. 1989. Description of a new species of net-winged midge (Diptera: Blepharicedidae) from the Sierra Nevada de Santa Marta, Colombia. Contributions in Sciences. Natural History Museum of Los Angeles County 340: 1-10.

Hogue, Ch. 1989. The net-wingued midge fauna (Diptera: Blephariceridae) of Antioquia Depatment, Colombia. Contributions in Science. 413: 1-14.

Hogue, Ch. 1990. A remarkable new species of Maruina (Diptera: Psychodidae) from Colombia. Aquatic Insects 12 (3): 185-191.

Holzenthal, R.W. 1988a. Studies in Neotropical Leptoceridae (Trichoptera), VIII: the genera Atanatolica Mosely and Grumichella Müller (Triplectidinae: Grumichellini). Transactions of the American Entomological Society 114: 71-128.

Holzenthal, R.W. 1988b. Systematic of Neotropical Triplectides (Trichoptera: Leptoceridae). Annals of the Entomological Society of America 81: 187-208.

Holzenthal, R.W. \& Blahnik, R.J. 1995. New species of Smicridea (Rhyacophylax) (Trichoptera: Hydropsychidae) from Costa Rica. Entomological News 106: 213-223. 
Holzenthal, R.W. y Flint, Jr. O.S. 1995. Studies of Neotropical caddisflies. LI: systematics of the Neotropical caddisfly genus Contulma (Trichoptera: Anomalopsychidae) Smithsonian Contributions to Zoology 575: 1-59

Hynes, H. B. N. 1959. The use of invertebrates as indicators of river pollution”. Porc. Linnean. Soc. London, (2): 165-170.

Hynes, H. B. N. 1963. The biology of polluted water. Liverpool University Press, 202 p.

Illies, J. \& L. Botosaneau. 1963. Problemès et mèthodes de la classification et dela zonation écologique des euax courants, considérées surtout du point de vue faunistique, Mitt. Int. Ver. Theor. Angew. Limnol., 12: 1-57.

Kolenati, F. A. 1848. Uber Nutzen und Schaden der Trichopteren”, Stettiner Entomol. Ztg. 9.

Kolkwitz, R \& Marsson, W.A. 1908. Ecology of plant saprobia. Verh. Ges. Oekol., 26: 505-519.

Kolkwitz, R \&Marsson, W.A. 1909. Ökologie der tierischen Saprobien. Beitäge zür Lehre von der biologische Gewäserbeuteilung. Internationale Reveu der gesamten Hydrobiologie, 2: 126-152.

Lizarralde de Grosso, M. 2009. En:Domínguez, E. y Fernández, H. (Eds). Macroivertebrados bentónicos sudamericanos. Sistemática y biología. Pp. 341-364Fundación Miguel Lillo. Tucumán, Argentina.

Longo, M. Ceballos, V.E. Zamora, G. \& Vásquez, G. 2004. Diversidad, similitud y carácter bioindicador de los macroinvertebrados acuáticos epicontinentales de tres ecosistemas lóticos en el piedemonte llanero. Unicauca Ciencia 8: 15-20.

Longo, M. Zamora, H., Vásquez, V.A. \& Velásquez, L.E. 2005. Aspectos ecológicos de Limnaea (Mollusca: Limnaeideae) en la región de Aguas Tibias, Municipio de PuracéCoconuco. Revista Asociación Colombiana de Ciencias Biológicas 17: 47-58.

Longo, M. \& Blanco, J.F. 2009. Sobre los filtros que determinan la distribución y abundancia de los macroinvertebrados diádromos y no-diádromos en cada nivel jerárquico del paisaje fluvial en islas. Actualidades Biológicas 31 (91): 179-195.

Longo, M., Gómez-Aguirre, A.M., Blanco, J.F. \& ZamoraGonzález, H. 2009. Cambios multianuales y espaciales de la composición y estructura del ensamble de insectos acuáticos en las quebradas perennes de la Isla Gorgona, Colombia. Actualidades Biológicas 31 (91): 141-160.

Longo, M., Zamora, H., Guisande, G. \& Ramírez, J.J. 2010. Dinámica de la comunidad de macroinvertebrados en la quebrada Potrerillos (Colombia): respuesta a los cambios estacionales de caudal. Limnetica 29 (2): 195-210.

Lorenz, C. M., G. M. van Dijk., A. G. M. van Hattum \& W. P. Cofino. 1997. Concepts in river ecology: implications for indicator development. Regul. Rivers: Res. Manage., 13: 501-516.

Lundblad, O. 1953. Die Hydracarinenfauna von Colombia. Ark. Zool. (ser. 2), 5 (8): 435-585
Machado, T. 1988. Distribución ecológica e identificación de los coleópteros acuáticos en diferentes pisos altitudinales del Departamento de Antioquia. Universidad de Antioquia, Medellín, Colombia.

Malek, E.A. \& Little, M.D. 1971.Aroapyrgus colombiensis n. sp. (Gastropoda, Hydrobiidae) snail intermediate host of Paragonimus caliensis in Colombia.Nautilus 85: 20-26.

Manzo, V. 2005. Key to the South America genera of Elmidae (Insecta: Coleoptera) with distributional data. Studies of Neotropical Fauna and Environmental 40 (3): 201-208.

Manzo, V. 2006. A review of the American species of XenelmisHinton (Coleoptera: Elmidae), with a new species from Argentina. Zootaxa 1242: 53-68.

Matthias, U. \& Moreno, H. 1983. Estudio de algunos parámetros físicoquímicos y biológicos del río Medellín y sus principales afluentes. Actualidades Biológicas 12 (46): 106-117.

Mazzucconi, S.A., López-Ruf, M.L. \& Bachmann, A.O. 2009. Hemiptera: Heteroptera: Gerromorpha y Nepomorpha.En: Domínguez, E. y Fernández, H. (Eds). Macroivertebrados bentónicos sudamericanos. Sistemática y biología.Pp. 167232. Fundación Miguel Lillo. Tucumán, Argentina.

Mendes, T., C. L. Elias, S. F. P. Almeida, A. R. Calapez \& M. J. Feio. 2014. Comparing alternatives for combining invertebrate and diatom assessment in stream quality classification. Marine and Freshwater Research 65 (7): 612-623.

Meza, A.M. Rubio, J. Días, L. \& Walteros, J. 2012. Calidad de agua y composición de macroinvertebrados acuáticos en la subcuenca alta del río Chinchiná. Caldasia 34 (2): 443-456.

Margalef, R. 1951. Diversidad de especies en las comunidades naturales. P. Inst. Biol. Appl. 9: 15-27.

Metcalf, J. L. 1989, "Biological water quality assessment of running waters based on macroinvertebrate communities: history and present status I, Europe”, Environ. Pollut., 60: 101-139.

Michaelsen, W. 1913-1914. Die Olichaeten Columbias, en: Fuhrmann and Mayr, Voyage d'exploration scientifique en Colombie. Mémoires de la Société Neuchâteloise des Sciences Naturelles 5: 202-252.

Molano, F. \& Camacho, D.L. 2006. Especies de Gerridae (Heteroptera:Gerromorpha) del Departamento del Quindío. Pp. 370-376. En: Molano, F. y Morales, I.T. (Eds.). Riqueza biótica quindiana. Universidad del Quindío, Armenia, Colombia.

Molano, F., Camacho, D.L. \& Serrato, C. 2005. Guerridae (Heteroptera: Gerromprpha) de Colombia. Biota Colombiana 6 (2): 163-172.

Molineri, C. 2010. A cladistic revision of Tortopus Needham \& Murphy with description of the new genus Tortopsis (Ephemeroptera: Polymitarcyidae). Zootaxa 2481: 1-36.

Molineri, C. \& Zúñiga, M. del C. 2004. Lumahyphes a new genus of Leptohyphidae (Insecta: Ephemeroptera). Aquatic Insects 26 (1): 19-30. 
Molineri, C. \& Zúñiga, M. del C. 2006. New species of Leptophyphidae (Insecta: Ephemeroptera) from Colombia with evidence of reproductive time segregation. Studies on Neotropical Fauna and Environment 41 (2): 139-151.

Molineri, C. Cuz, P.V. \& Emerich, D. 2011. A new species of Asthenopus (Ephemeroptera: Polymitarcyidae: Asthenopodinae) from Brasil and Colombia. Zootaxa 2750: 33-38.

Molineri, C., Peters, J. \& Zúñiga, M. del C. 2002. A new family, Coryphoridae (Ephemeroptera: Ephemerelloidea) and description of the winged and egg stages of Coryphorus. Insecta Mundi 15 (2): 117-122.

Montes-Fontalvo, J.M. \& Pérez-Gutiérrez, L.A. 2011a. Rediscovery of Mesagrion leucorrhinum (Zygoptera: Megapodagrionidae): a formal description of female and ultimate stadium of larva with notes on habits. International Journal of Odonatology 14 (1): 91-100.

Montes-Fontalvo, J.M. y Pérez-Gutiérrez, L.A. 2011b. Heteropodagrion croizati sp. nov. (Odonata: Megapodagrionidae) with a key to the known species of the genus.Zootaxa 2810: 63-68.

Monsalve, C. A. 2004. Palinología del Holoceno superior en la laguna Puente Largo, Páramo de Frontino, Antioquia, cordillera Occidental, Colombiana. Tesis Magíster, Universidad Nacional de Colombia, Facultad de Ciencias Agropecuarias. Medellín.

Morales-Castaño, I.T. \& Molano-Rendón, F. 2008. Heterópteros acuáticos del Quindio (Colombia): Los infraórdenes Gerromorpha y Nepomorpha. Revista Colombiana de Entomología. 34 (1): 121-128.

Mosquera, S. Alba-Tercedor, J. \& Zúñiga, M. del C. 2001. Atopophlebia fortunensis Flowers (Ephemeroptera: Leptophlebiidae) and Caenis chamie (Ephemeroptera: Caenidae). Notes on their biology and ecology.Pp. 157160, En: Domínguez, E. (Ed.), Trends in Research in Ephemeroptera and Plecoptera. Kluwer Academic Plenum Publishers. New York, USA.

Muñoz, D. \& Ospina, R. 1999.Guía para la identificación genérica de los Ephemeroptera de la Sabana de Bogotá, Colombia. Ninfas y algunos géneros de adultos. Actualidades Biológicas 21 (70): 47-60.

Muñoz, F. 2000. Especies del orden Trichoptera (Insecta) en Colombia. Biota Colombiana 1 (3): 267-288.

Muñoz, F. 2004. El Orden Trichoptera (Insecta) en Colombia, II: inmaduros y adultos, consideraciones generales. Pp. 319-349. En: Fernández, F., Andrade, M.G., y Amat, G. (Eds.). Insectos de Colombia No 3. Universidad Nacional de Colombia, Facultad de Ciencias. Santafé de Bogotá, Colombia.

Muñoz, M \& Vélez, I. 2007. Redescripción y algunos aspectos ecológicos de Girardia tigrina, G. cameliae y G. paramensis (Dugesiidae, Tricladida) en Antioquia, Colombia. Revista Mexicana de Biodiversidad 78: 291- 301, 2007

Munné, A. C. Solà, \& Prat, N. 1998. QBR: un índice rápido para la evaluación de la calidad de los ecosistemas de ribera. Tecnología del agua, 175: 20-37.
Muñoz-Quesada, F. 1997. Five new species and a new record of Costa Rican Leptonema Guérin (Trichoptera: Hydropsychidae). Proceeding of the Entomological Society of Washington 99: 115.

Navas, L. 1935. Odonatos de Colombia. Boletín de la Sociedad Entomológica Española. 17: 33-38.

Nazarova, L.B., Riss, W., Kahlheber, A. \& Werding, B. 2004. Some observations of buccal deformities in chironomid larvae (Diptera: Chironomidae) from the Ciénaga Grande de Santa Marta, Colombia. Caldasia 26 (1): 275-290.

Novelo-Gutiérrez, R. 1995a. La náyade de Brechmorhoga praecox (Hagen, 1861) y notas sobre las náyades de $B$. rapax Calvert, 1898, B. vivax Calvert, 1906 y B. mendax (Hagen, 1861) (Odonata: Libellulidae). Folia entomológica mexicana 94: 33-40.

Novelo-Gutiérrez, R. 1995b. Náyade de Brechmorhoga pertinax (Odonata: Libellulidae). Anales del Instituto de Biología Universidad Nacional Autónoma de México, serie Zoología 66 (2): 181-187.

Ospina, T.R. Riss, W. \& Ruíz, J.L. 1999. Guía para la identificación genérica de larvas de quironómidos (Diptera: Chironomidae: Orthocladiinae) de la Sabana de Bogotá. Pp. 363-383, En: Amat, G., Andrade, M.G. y Fernández, F. (Eds.), Insectos de Colombia No 2. Revista de la Academia Colombiana de Ciencias Exactas Físicas y Naturales. Colección Jorge Alvárez Lleras No 13. Santafé de Bogotá, Colombia.

Padilla-Gil, D.N. \& Nieser, N. 2003. Nueva especie de Tachygerris y nuevos registros de colecta de los Gerridae (Hemiptera: Heteroptera) de Colombia. Agronomía Colombiana 21 (1-2): 51-57.

Padilla-Gil, D.N. 2010a. Five new species of Buenoa (Hemiptera: Heteroptera: Notonectidae) from Colombia. Zootaxa 2411: 22-32.

Padilla-Gil, D.N. 2010b. Two new species of Martarega (Hemiptera: Heteroptera: Notonectidae) and a new species of Tachygerris from Colombia. Zootaxa 2560: 61-68.

Padilla-Gil, D.N. 2012a. Two new species of Rhagovelia from Colombia (Hemiptera:Heteroptera: Veliidae) with revised keys to the Colombian species in the torquata and robusta groups. Zootaxa 325: 57-63

Padilla-Gil, D.N. 2012b. Los hemípteros acuáticos del Municipio de Tumaco (Nariño, Colombia). Guía ilustrada. Editorial Universitaria-Universidad de Nariño, San Juan de Pasto.

Padilla, D.N. 2013. Description of the egg and immature stages of Potamobates anchicaya J. Polhemus \& D. Polhemus, 1995 (Hemiptera: Heteroptera: Gerridae) and intersexual variation in adults”. Zootaxa. 5: 524-532.

Padilla, D.N, y F. Ferraz. 2013. Two new species of Microvelia, 1834 (Hemiptera: Heteroptera: Veliidae) from Colombia, with a key to Colombian species. Zootaxa. 5: 587-595.

Padilla, D.N. 2014. New records of aquatics Heteroptera (Hemiptera) from the Andean foothills of the Amazonia (Putumayo, Colombia). Revista Colombiana de Entomologia. 40 (2): 234-238. 
Padilla, D.N. 2014. Distribución espacial de las especies del género Buenoa Kirkaldy 1904 (Hemiptera: Notonectidae) en Tumaco (Nariño, Colombia). Acta Biológica Colombiana. 19 (1): 83-88.

Padilla, D.N. 2015. Gerromorpha y Nepomorpha (Heteroptera) del Pacífico de Colombia; lista de especies, distribución geográfica y altitudinal. Biota Colombiana. 16 (1): 20-35.

Padilla, D.N. 2015. Description of the egg and immature stages of Martarega lofoides Padilla-Gil, 2010 (Hemiptera: Heteroptera: Notonectidae). Zootaxa.4: 593-599.

Padilla, D.N. 2015. Ten new species of Rhagovelia in the angustipes complex (Hemiptera: Heteroptera: Veliidae) from Colombia, with a key to the Colombian species. Zootaxa. (1): 71-95.

Padilla, D.N. 2016. Las chinches semi-acuáticas de la Reserva Natural Río Ñambí (Nariño), Colombia. Acta Biologica Colombiana. 21 (1): 201-206.

Paggi, A.C. 1999. Los Chironomidae como indicadores de calidad de ambientes dulceacuícolas. Revista de la Sociedad Entomológica Argentina 58: 202-207.

Palacino-Rodríguez, F. 2009. Dragonflies (Anisoptera: Odonata) of the collection of the Instituto de Ciencias Naturales Universidad Nacional de Colombia. Boletín del Museo de Entomología de la Universidad del Valle. 10 (1): 37-41.

Parra- Trujillo, Y.T, D. N. Padilla y G. Rreinoso. 2014. Diversidad y distribución de Rhagovelia (Hemiptera, Veliidae) del departamento del Tolima”. Revista de la Asociación Colombiana De Ciencias Biológicas. 26: 82-88.

Patiño-González J. (Hermano Daniel). 1941 Apuntes sobre algunos moluscos colombianos. Revista de la Academia de Ciencias Exactas Físicas y Naturales 4: 15-16.

Patrick, R. 1949. A proposed biological measure of stream conditions, based on a survey of the Conestoga basin, Lacaster County, Pennsylvania. Proc. Acad. Nat. Sci. Phila., 101: 277-341.

Patrick, R. 1950. Biological measure of stream conditions.Sewage ind. Wastes. 22: 926-939.

Pérez, G. \& Roldán, G. 1978. Niveles de contaminación por detergentes y su influencia en las comunidades bénticas del Río Rionegro (Antioquia). Actualidades Biológicas 7 (24): 27-36.

Pérez, L.A. \& Gutiérrez. 2003. Estudio biotaxonómico de los odonatos (Insecta: Odonata Fabricius, 1973) del Distrito de Santa Marta (Magdalena, Colombia). Tesis de pregrado. Universidad del Magdalena, Facultad de Ciencias Básicas, Programa de Biología. Santa Marta, Colombia.

Pérez-Gutiérrez, L.A. 2007. The larvae of Teinopodagrion caquetanum De Marmels and T. vallenatum De Marmels (Zygoptera: Megapodagrionidae). Odonatologica 36 (3): 307-313.

Pérez-Gutiérrez, L.A., Monroy-González, J.D. \& RealpeRebolledo, E.A. 2007. Local assemblage patterns of odonates in Central Chocó, Colombian Pacific Pp 183-199. En: India (Ed.). Odonata - Biology of dragonflies.
Perla Alonso-Eguíalis, Mora, J.M. Campbel. B\& Springer, M. (Eds). 2014. Diversidad, conservación y uso de los macroinvertebrados dulceacuícolas de México, Centroamérica, Colombia, Cuba y Puerto Rico. IMTA México.

Pilsbry, H.A. 1955. South American land and freshwater mollusks. IX Colombian Species Lymnaeabogotensis.Proceeding of the Academy of Natural Sciences of Philadelphia 87: 83-88.

Polhemus, J.T. \& Manzano, M.R. 1992. Marine Heteroptera of the Eastern Tropical Pacific (Gelastocoridae, Gerridae, Mesoveliidae, Saldidae, Veliidae).Pp. 302-320.En: Quintero, D. y Avello, A. (Eds.). Insects of Panamá and Mesoamérica. Oxford University Press.

Polhemus, J.T. \& Polhemus, D.A. 1995. A phylogenetic review of the Potamobates fauna of Colombia (Hemiptera: Gerridae) with descriptions on three new species. Proceeding of the Biological Society of Washington 97 (2): 350-372.

Posada, J. A. \& Roldán, G. 2003. Clave ilustrada y diversidad de las larvas de Trichoptera en el nor-occidente de Colombia. Caldasia 25 (1): 169-192.

Posada-García, J.A., Abril-Ramírez, G. y Parra-Sánchez, L.N. 2008. Diversidad de los macroinvertebrados acuáticos del páramo de Frontino (Antioquia, Colombia). Caldasia 30 (2): 441-445.

Posso, C.E. \& González, R. 2008. Gerridae (Hemiptera: Heteroptera) del Museo Entomológico de la Universidad del Valle.Revista Colombiana de Entomología 34 (2): 230-238.

Prain, T. 1956. On a collection of Pomacea from Colombia, with description of a new subspecies.Journal of Conchology 24: 73-79.

Quintero, A. \& Rojas, A.M. 1987. Aspectos bioecológicos del orden Trichoptera y su relación con la calidad del agua. Revista Colombiana de Entomología 13 (1): 26-38.

Quiñonez, M., Ramírez, J. \& Días, A. 1998. Estructura numérica de la comunidad de macroinvertebrados acuáticos derivadores en la zona de ritral del río Medellín. Actualidades Biológicas 20: 7586.

Ramírez, A. 1996. Six new dragonfly larvae of the family Gomphidae in Costa Rica, with a key to the Central American genera (Anisoptera). Odonatologica 25 (2): 143-156.

Ramírez, A y Novelo-Gutiérrez, R. 1999. The Neotropical dragonfly genus Macrothemis: new larval descriptions and an evaluation of its generic status based on larval stage (Odonata: Libellulidae). Journal of the North American Benthological Society 18 (1): 67-73.

Ramos, A. 1997. Coleópteros acuáticos y semiacuáticos en cuatro ríos del sur occidente colombiano. Tesis de pregrado. Universidad del Valle, Programa de Biología. Santiago de Cali, Colombia.

Realpe, E. 2009. Diversidad del generoIschnura (Odonata: Coenagronidae) y su relación con la altitud y orogenia de la Cordillera Oriental-Andes colombianos. Tesis de doctorado. Universidad de los Andes. 
Reinoso-Flórez, G. 1999. Estudio de la fauna béntica del río Combeima, Colombia. Revista de la Asociación Colombiana de Ciencias Biológicas, Vol: 11 Fasc: 1 pp: 35-44.

Reinoso, G., Guevara, G., Arias, D., García, J \&Villa, F. 2007. Aspectos bioecológicos de la fauna entomológica de la cuenca mayor del río Coello - Departamento del Tolima, Colombia. Revista de la Asociación Colombiana de Ciencias Biológicas 19 (1):65-72.

Reinoso, G., Guevara, G. Vejarano, M., García, J \& Villa, F. 2008. Evaluación del río Prado a partir de los macroinvertebrados y de la calidad del agua. Revista de la Asociación Colombiana de Ciencias Biológicas 20 (1): 102-116.

Resh, V. H., Richard, H. N \&. Barbour, M. T. 1995. Design and implementation of rapid assessment approaches for water resource monitoring using macroinvertebrates. Aust. J. Ecology., 20: 108-121.

Rincón, M. E. 2002. Comunidad de insectos acuáticos de la quebrada Mamarramos (Boyacá, Colombia). Revista Colombiana de Entomología 28 (1): 101-108.

Rincón, M.E. 1996. Aspectos bioecológicos de los tricópteros de la quebrada Carrizal (Boyacá, Colombia). Revista Colombiana de Entomología 22: 53-60.

Rincón, M.E. \& Castro, M.I. 2008. Efecto del caudal sobre la emergencia de Trichoptera.Pp. 211-223. En: Donato, J.(Ed.) Ecología de un río de montaña de los AndesColombianos (Río Tota, Boyacá). Universidad Nacional de Colombia, Facultad de Ciencias. Bogotá, Colombia.

Rincón, M.E. 1999. Estudio preliminar de la distribución altitudinal y espacial de los tricópteros en la cordillera Oriental, Colombia. Pp. 267-282. En: Andrade, M.G., Amat, G. y Fernández, F. (Eds.), Insectos de Colombia No 2. Academia Colombiana de Ciencias Exactas, Físicas y Naturales. Santafé de Bogotá.

Ringuelet, R.A. 1972. Hirudíneos neotropicales de Colombia, Cuba y Chile, con descripción de una nueva especie de Oligobdella (Glossiphoniidae). Physis 31 (83): 345-352.

Ringuelet, R.A. 1974. Los hirudíneos terrestres del género Blanchiardella Weber del Páramo norandino de Colombia. Physis 33 (86): 63-69.

Ringuelet, R.A. 1975. Un nuevo hirudíneo de Colombia parásito de la trucha arco iris. Neotropica 21 (64): 1-4.

Riss, W., Ospina, R. \& Gutiérrez, J.D. (2002). Establecimiento de valores de Bioindicación para macroinvertebertados acuáticos de la Sabana de Bogotá. Caldasia 24 (1): 135-156.

Roback, S.S. \& Nieser, N. 1974. Aquatic Hemiptera (Heteroptera) from the Llanos Orientales of Colombia.Proceedings of the Academy of Natural Sciences of Philadelphia 126 (4): 29-49.

Rocha, C.M. 1994. Diversidad en Colombia de los cangrejos del género Neostrengeria. Academia Colombiana de Ciencias Exactas, Físicas y Naturales. Colección Jorge Arbeláez Lleras No. 5. 143p.
Rodríguez-Barrios, J., Ospina-Torres, R, Gutiérrez, J. D\& Ovalle, H. 2007. Densidad y biomasa de macroinvertebrados acuáticos derivantes en una quebrada tropical de montaña (Bogotá, Colombia). Caldasia 29 (2): 397-412.

Rodríguez-Barrios, J., Ospina-Torres, R. \& Turizo-Correa, R. 2011. Grupos funcionales alimentarios de macroinvertebrados acuáticos en el río Gaira, Colombia. Revista de Biología Tropical. 59 (4): 1537-1552.

Rodríguez, F. 2009. Dragonflies (Odonata: Anisoptera) of the collection of the Instituto de Ciencias Naturales, Universidad Nacional de Colombia. Boletín del Museo de Entomología de la Universidad del Valle 10 (1): 37-41.

Rodríguez,G. 1972.Trichodactylidae from Venezuela, Colombia, andEcuador (Crustacea: Brachyura Alfre D E. Smalley Department of Biology, Tulane University, New Orleans, Louisiana 70118 and Reprinted from Tulane Studies in Zoology and Botany, Vol. 17, No. 3. p. 41-55 .

Rodriguez, G. 1981. Parte I; Arthropoda en Hulbert, S.H., G. Rodriguez y N.d. Sabtos (Ed). Aquatic biota of tropical South America. San Diego State University, San Diego, California. 223pp.

Rodríguez, G. 1985. A new cavernicolous crab (Crustacea, Decapoda, Pseudothelphusidae) from Colombia. 33. 2 (7380) (EnglM 20 ref., 3 figM 1 tab.)- Instituto Venezolano de Investigaciones Científicas, Caracas 1010-A, Venezuela.

Rojas, A.M. \& Baena, M.L. 1993. Anacroneuria farallonensis (Plecoptera: Perlidae) una nueva especie para Colombia. Boletín del Museo de Entomología de la Universidad del Valle 1: 23-28.

Rojas, A.M. \& Zúñiga, M. del C. 1996. Aspectos bioecológicos del orden Ephemeroptera en cuencas de algunos ríos del Departamento del Valle del Cauca (Colombia). Pp. 453-472. En: Andrade, G., Amat, G. y Fernández, F. (Eds.). Insectos de Colombia. Estudios escogidos. Academia Colombiana de Ciencias Exactas Físicas y Naturales. Colección Jorge Álvarez Lleras No. 10.

Rojas, A.M. Zuñiga, M. del C., Burbano, M.E. \&Serrato, C. 1995. Altitudinal distribution of Ephemeroptera in the Farollones de Cali National Park, Colombia.Pp. 121-129. En: Corkum, L.P. y Ciborowski, J.J.N. (Eds.).Current directions in research on Ephemeroptera.Canadian Scholars Press, Inc. Toronto, Canadá.

Rojas-Riaño, N.C. 2011. First record of the damselfly genus Anisagrion (Odonata: Coenagrionidae) from Colombia. Revista Colombiana de Entomología 37 (1): 164-165.

Roldán, G., Builes, J. J., Trujillo, C. M. \& Suárez, A. 1973. Efectos de la contaminación industrial y doméstica sobre la fauna béntica del río Medellín. Actualidades Biológicas 2 (5): 54-64.

Roldán, G. 1980. Estudio limnológico de cuatro ecosistemas neotropicales diferentes con especial referencia a su fauna de efemerópteros. Actualidades Biológicas 9 (34): 103-117.

Roldán, G. 1985. Contribución al conocimiento de las ninfas de efemerópteros en el Departamento de Antioquia. Actualidades Biológicas 14 (51): 3-13. 
Roldán, G. 1988. Guía para el estudio de los macroinvertebrados acuáticos del Departamento de Antioquia. Fondo FENColombia- Conciencias - Universidad de Antioquia, Editorial Presencia Ltda. Santafé de Bogotá.

Roldán, G. 1999. Los macroinvertebrados y su valor como indicadores de la calidad del agua. Revista de la Academia Colombiana de Ciencias Exactas Físicas y Naturales 23 (88): 375-387.

Roldán, G. 2001. Estudio limnológico de los recursos hídricos del parque de Piedras Blancas. Academia Colombiana de Ciencias Exactas Físicas y Naturales Colección Jorge Álvarez Lleras No. 9.

Roldán, G. 2003. La bioindicacion de la calidad del agua en Colombia. Editorial Universidad del Antioquia, Medellín. 170p.

Roldán, G, Zúñiga, M. del C, Zamora, H, Álvarez, L.F. Reinoso, G. \& M. Longo, M. 2014. Capitulo de Colombia. En: Perla Alonso-Eguíalis, José Manuel Mora, Bruce Campbell y Monika Springer, Editores 2014. Diversidad, conservación y uso de los macroinvertebrados dulceacuícolas de México, Centroamérica, Colombia, Cuba y Puerto Rico. IMTA México.

Romero, I.R., Pérez, S.M. \& Rincón, M.E. 2006. Aspectos ecológicos de los Trichoptera del Parque Nacional Natural Cueva de los Guácharos, Huila (Colombia). Actualidad y Divulgación Científica 9: 129-140.

Romero, F. \& Navarro, F. 2009. Lepidoptera. En:Domínguez, E. y Fernández, H. (Eds.). Macroinvertebrados bentónicos sudamericanos: Sistemática y biología.Pp. 309-340. Primera Edición. Fundación Miguel Lillo, San Miguel de Tucumán, Argentina.

Rosso de Ferradás, B. \& Fernández, H.R. 1995. Acari, Hydrachnidia. Pp. 819-853. En: Loreto, E. y Tell, G. (Eds.). Ecosistemas de aguas continentales. Editorial Sur, Argentina.

Rosso de Ferradás, B. \& Fernández, H.R. 2005. Elenco y biogeografía de los ácaros acuáticos (Acari, Parasitengona, Hydrachnidia) de Sudamérica. Graellsia 61: 181-224.

Rosso de Ferradás, B. \& Fernández, H.R. 2009. Acari, Parasitengona, Hydrachnidia.. En: Domínguez, E. y Fernández, H. (Eds). Macroivertebrados bentónicos sudamericanos. Sistemática y biología.Pp 497-550. Fundación Miguel Lillo. Tucumán, Argentina.

Rúa-García, G. A. 2012. Distribución y composición de los órdenes Ephemeroptera, Plecoptera y Trichoptera en cuatro ríos de la Sierra Nevada de Santa Marta, Colombia. Tesis de pregrado. Universidad del Magdalena. Programa de Biología, Santa Marta.

Rueda-Delgado, G. (Ed.). 2002. Manual de Métodos en Limnología. Asociación Colombiana de Limnología. Pen Clips Publicidad y Diseño Ltda. Santafé de Bogotá.

Ruíz, J., Ospina, R. \& Riss, W. 2000a. Guía para la identificación genérica de larvas de quironómidos (Diptera: Chironomidae) de la Sabana de Bogotá. II. Subfamilia Chironominae. Caldasia 22 (1): 15-33.
Ruíz, J., Ospina, R., Gómez, H. \& Riss, W. 2000b. Guía para la identificación genérica de larvas de quironómidos (Diptera: Chironomidae) de la Sabana de Bogotá. III. Subfamilias Tanypodinae, Podonominae y Diamesinae. Caldasia 22 (1): 34-60.

Salinas, L.G., Dias, L., Salles, F. \& Bacca T. 2011. Three new species of Baetodes Needham \& Murphy (Ephemeroptera: Baetidae) from Colombia. Zootaxa 3110: 61-68.

Sánchez-Herrera, M.J. 2005. El índice biológico BMWP (Biological Monitoring Working Party score), modificado y adaptado al cauce principal del río Pamplonita, Norte de Santanter. Bistua 3 (2): 54-67.

Sánchez-Herrera, M.J. \& Vendaño-Sánchez, M. 2005. Macroinvertebrados del Norte de Santander. Respuestas, Revista de la Universidad Francisco de Paula Santander 1: 3-20.

Serna, D. 2003. Estructura de la comunidad de larvas del orden Trichoptera y su dinámica espacio-temporal en un gradiente altitudinal de la cuenca del río Manzanares, Santa Marta, Caribe colombiano. Tesis de Pregrado. Facultad de Ciencias Básicas. Universidad del Magdalena.

Serna-Zamora, M.Y. \& Zamora, H. G. 2004. Biogeografía y relaciones de similitud entre laspoblaciones de macroinvertebrados acuáticos epicontinentales en un transecto Cauca-Meta. Revista de la Asociación Colombiana de Ciencias Biológicas 16 (1): 71-79.

Shannon, C. E. \&Weaver, W. 1949. The mathematical theory of communication.The Universisity of Illinois Press, Urbana, Illinois, pp 19-27, 82-103, 104-107.

Sites, R. \& Álvarez, L.F. 2010. Description of the female of Procryphocricos perplexus Polhemus (Hemiptera: Heteroptera: Naucoridae). Zootaxa 2562: 66-68.

Sládeček, V. 1962. A guide to limnosaprobical organisms. Sci. Pap. Inst. Chem. Technol. Water, 7 (2): 543-612.

Soler, E. 1983. Contribución al estudio taxonómico y ecológico de caracoles (pulmonados de agua dulce) de la Sabana de Bogotá. Tesis de pregrado. Universidad Nacional de Colombia. Programa de Biología. Santafé de Bogotá.

Spangler, P.J. \& Santiago-Fragoso, S. 1987. A revision of the genera Disersus, Pseudodisersus and Potamophilops of the Western hemisphere (Coleoptera: Elmidae) Smithsonian Contributions to Zoology No 446. Smithsonian Institution Press, Washington.

Spangler, P.J. \& Santiago-Fragoso, S. 1992. The aquatic beetle subfamily Larainae (Coleoptera: Elmidae) in México, Central America and West Indies. Smithsonian Contributions to Zoology No 528. Smithsonian Institution Press, Washington.

Spangler, P.L. 1981. Coleoptera. En: Hulbert, S.H., Rodríguez, G y Santos, D. (Eds.). Aquatic Biota of Tropical South America.Part 1.Pp. 129-220. San Diego State University. San Diego, California.

Springer, M., Ramírez,A. \& Hanson. P. 2010. Macroinvertebrados de Agua dulce de Costa Rica I. Revista de Biología Tropical, 58 (Supl. 4). 
Suárez, M. 1987. Contribución al estudio de los odonatos del Valle del Cauca. Tesis de grado. Universidad del Valle. Cali. Colombia.

Stark, B.P., Froehlich, C. \& Zúñiga, M. del C. 2009. South American stoneflies (Plecopera) En: Adis, J., Arias, J.R., Golovatch, S., Wantzen, K.M. y Rueda-Delgado, G. (Eds.). Aquatic Biodiversity of Latin America (ABLA), Vol 5. Pensoft, Sofia-Moscow.

Stark, B.P., Zúñiga, M. del C., Rojas, A.M. \& Baena, M.L. 1999. Colombian Anacroneuria: descriptions of new and old species (Insecta: Plecoptera: Perlidae). Spixiana 22: 13-46.

Stark, P.B. \& Zúñiga, M. del C. 2003. The Anacroneuria guambiana complex of South America (Plecoptera: Perlidae). Pp. 151-159. En: Gaino, E. (Ed.). Research update on Ephemeroptera and Plecoptera. University of Perugia, Italy.

Stark, P.B. Zúñiga, M. del C. \& Sivec, I. 2002. Description of Anacroneuria (Plecoptera: Perlidae) from the upper Río Amazonas drainage, Colombia and Perú. Acta Entomologica Slovenica 9 (2): 119-122.

Statzner, B., B. Bis, S. Dolédec \& P. Usseglio-Polatera. 2001. Perspectives for biomonitoring at large spatial scales: a unified measure for the functional composition of invertebrate communities in European running waters. Basic and Applied Ecology 2: 73-85.

Suárez, M. 1987. Contribución al estudio de los odonatos del Valle del Cauca. Tesis de pregrado. Universidad del Valle. Programa de Biología. Santiago de Cali.

Tamaris-Turizo, C. 2009. Transporte de materia orgánica y deriva de macroinvertebrados acuáticos a lo largo de un río tropical. Tesis de Maestría en Acuicultura y Ecología Acuática Tropical. Instituto de Posgrados. Universidad del Magdalena.

Tamaris-Turizo, C. \& Sierra-Labastidas, T. 2009. Una inspección al papel de la producción de los Plecopteros en los sistemas lóticos. Revista de Ciencias. Facultad de Ciencias Naturales y Exactas de la Universidad del Valle. Vol. 13: 109-120

Tamaris-Turizo, C., Turizo, R. \& Zúñiga, M. del C. 2007. Distribución espacio-temporal y hábitos alimentarios de ninfas de Anacroneuria (Insecta:Plecoptera:Perlidae) en el río Gaira (Sierra Nevada de Santa Marta, Colombia). Caldasia 29 (2): 375-385.

Towsend, C. R. \& M. R. Scarsbrook. 1997. Quantifying disturbance in streams: alternative measures of disturbance in relation to macroinvertebrate species traits and species richness. J. N. Am. Benthol. Soc., 16 (3): 531-544.

Trihadiningrum, Y., N. De Pauw, I. Tjondronegoro \& R. F. Verheyen. 1996, Use of benthic macroinvertebrates for quality assessment of the Blawi river (East Java, Indonesia), en: Perspectives in tropical limnology, F. Schiemer y K. T. Boland, eds., SPB Academic Publishing, Amsterdam.

Urrutia, M.X. 2005. Riqueza de especies de Odonata Zigoptera por unidades fisiográficas en el Departamento del Valle del Cauca. Boletín del Museo de Entomología de la Universidad del Valle 6 (2): 30-36.

Valencia, D\& Campos, M. 2007. Freshwater prawns of the genus Macrobrachium Bate, 1868 (Crustacea: Decapoda: Palaemonidae) of Colombia. Zootaxa 1456: 1-44 (2007).

Vásquez, J.M., Ramírez, F. Guevara G \& Reinoso, G. 2010. Distribución espacial y temporal de los tricópteros inmaduros de la cuenca del Río Totaré (Tolima, Colombia). Caldasia 32 (1): 129-148.

Vásquez-Ramos, M. y Reinos-Flórez, G. 2012. Estructura de la fauna béntica en corrientes de los Andes colombianos. Revista Colombiana de Entomología 38 (2): 351-358.

Vásquez, J.M. Guevara G \& Reinoso, G. 2013. Impactos de la urbanización y agricultura en cuencas con bosque seco tropical: influencia sobre la composición y estructura de larvas de tricópteros. Rev. Asoc. Col. Cienc. Biol.(Col.), 25: 61-70.

Vásquez, J.M. Guevara G \& Reinoso, G. 2014. Factores ambientales asociados con la preferencia de habitat de larvas de tricopteros en cuencas con bosque seco tropical (Tolima, Colombia). Rev. Biol. Trop. (Int. J. Trop. Biol. 7744) Vol. 62 (Suppl. 2): 21- 40.

Viets, K. 1956. Wassermilben (Hydrachnellae, Acari) aus Venezuela und Kolumbien Pp. 315-327. En: Ergebnisse der deutschen limnologischen Venezuela-Expedition 1952. Band 1. Deutscher Verlag der Wissenschaften, Berlin.

von Ellenrieder, N. \& Garrison, R.W. 2009. Odonata. En: Domínguez, E. y Fernández, H. (Eds.). Macroinvertebrados bentónicos sudamericanos: Sistemática y biología. Pp. 96143. Primera Edición. Fundación Miguel Lillo, San Miguel de Tucumán, Argentina.

von Prahl, H. 1988. Cangrejos de agua dulce (Crustacea, Brachyura, Pseudothelphusidae y Trichodactylidae) capturados en el Departamento de Antioquia, Colombia. Boletín. Ecotrópica 18: 3-11.

Walter, C. 1914. Hydracarina de Colombie. En: Fuhrmann, O. y Mayor, E. Voyage d'exploration scientifique en Colombie. Pp. 193-201. Mémoires de la Société Neuchâteloise des Sciences Naturelles. Vol. 5. Neuchatel, Attinger.

Watling, L. 2003. A new species of Hyalella from Colombia, and the redescription of $\mathrm{H}$. meinerti Stebbing, 1899 from Venezuela (Crustacea: Amphipoda). Journal of Natural History, 37 (17), 2095-2111.

Washington, H. G. 1984. Diversity, biotic and similarity indices. Water Res., 18 (6): 653-694.

Wayne, N. \& Hogue, Ch. 1986. Description of the new species of the shore fly genus Driedrops (Diptera; Ephydridae) from Colombia. Contributions in Sciences-Natural History Museum of Los Angeles County 377: 21-26.

Weber, M. 1913. Hirudinéens Colombiennes. Mémoires de la Société Neuchâteloise des Sciences Naturelles 5: 731-747.

Welkenson, R.C. 1979. Horse-flies (Diptera: Tabanidae) of the Colombian Departments of Chocó, Valle, and Cauca. Cespedecia 8 (31-32): 87-435. 
Wiedenbrug, S. \& Ospina-Torres, R. 2005. A key to pupal exuviae of Neotropical Tanytarsini (Diptera: Chironomidae). Amazoniana 18 (3-4): 317-371.

Wilhm, J. F. y Dorris, T.C. 1968. Biological parameters of water quality.Bioscience 18: 447- 481.

Wilhm, J. L. 1967. Comparison of some diversity indices applied to populations of benthic macroinvertebrates in a stream receiving organic wastes. J. Wat. Pollut. Control. Fed., 39: 1673-1683.

Wilhm, J. L., \& Dorris, T. C. 1966. Species diversity of benthic macroinvertebrates in a stream receiving domestic and oil refinery effluents. Am. Midl. Nat., 76: 427- 449.

Wilhm, J. L., \& T. C. Dorris. 1968. Biological parameters for water quality criteria. Bioscience, 18: 447-481.

Williamson, E.B. 1918a. A collecting trip to Colombia, South America.Miscellaneous Publications.Museum of Zoology of Michigan 3: 1-24.

Williamson, E.B. 1918b. Results of the University of MichiganWilliamson expedition to Colombia, 1916-1917: two interesting new Colombian Gomphines (Odonata). Occasional papers of the Museum pf Zoology of Michigan 52: 1-14.

Williamson, E.B. 1919. Results of the University of MichiganWilliamson Expedition to Colombia, 1916-1917: notes on species of the genus Heteragrion Selys with descriptions of new species (Odonata). Occasional papers of the Museum of Zoology of Michigan. 68: 1-89.

Williamson, E.B. 1920. Results of the University of MichiganWilliamson Expedition to Colombia, 1916-1917: Notes on species of the genus Progomphus (Odonata). Occasional papers of the Museum of Zoology of Michigan No 77: 1-21.

Wooldride, D. 1973. New Paracymus from South America. Journal of Entomological Society 46: 116-123.

Wooldridge, D. 1976. New world Limnichidae. VI. A revision of Limnichoderus Casey (Coleoptera: Limnichidae). Journal of the Kansas Entomological Society 11: 163-173.

Zamora, H. 1995. Relaciones de similitud entre comunidades de macroinvertebrados dulceacuícolas de ecosistemas lénticos, ubicados entre la costa pacífica caucana y el piedemonte amazónico. Revista de la Asociación Colombiana de Ciencias Biológicas 9 (1-2): 7-21.

Zamora, H. 1996. Aspectos bioecológicos de las comunidades de macroinvertebrados dulceacuícolas en el Departamento del Cauca. Unicauca Ciencia 1: 1-11.

Zamora, H. 2000. Adaptación del índice BMWP para la evaluación biológica de la calidad de las aguas epicontinentales en Colombia. Unicauca Ciencia 4: 47-59.

Zamora, H. 2002. Análisis biogeográfico de los macroinvertebrados acuáticos epicontinentales (MAE) en el Departamento del Cauca, Colombia. Revista de la Asociación Colombiana de Ciencias Biológicas 14 (1): 37-64.

Zamora, H. 2010. Importancia de los macroinvertebrados acuáticos epicontinentales en la dieta natural de tres especies de peces nativos del río Patía, departamento del Cauca. Unicauca Ciencia (13): 65-75.

Zamora, H. \& Roessler, E. 1995. Descripción morfológica y taxonómica de una nueva especie de Anacroneuria (Insecta-Plecoptera). Revista de la Asociación Colombiana de Ciencias Biológicas 9 (1-2): 85-91.

Zamora, H. y Roessler, E. 1997. Número de estadios nayadales, ciclo biológico y patrón de crecimiento de Anacroneuria caucana (Insecta: Plecoptera). Unicauca Ciencia 2: 15-24.

Zamora, H., Naundorf, G. \& Vásquez, G.L. 1996. Macroinvertebrados dulceacuícolas del Parque Natural Nacional Isla Gorgona, Departamento del Cauca. Unicauca Ciencia 1: 12-18.

Zamora, H. \& Sarria, H. 2001. Calidad biológica de dos ecosistemas lóticos afectados por aguas residuales de rallanderías de yuca mediante la utilización de sus macroinvertebrados acuáticos como bioindicadores, comparando además la aplicación de los índices de Shannon-Weaver y BMWP. Unicauca Ciencia 6: 21-42.

Zúñiga, M. del C. 2009. Bioindicadores de calidad de agua y caudal ambiental.: Caso del Río Meléndez (Valle del Cauca, Colombia). Pp. 303-310. En: Cantera, J., Y. Carvajal y L. Castro (Compiladores). Caudal ambiental: Conceptos Experiencias y Desafíos. Programa Editorial de la Universidad del Valle, Cali, Colombia.

Zúñiga, M. del C. 2010. Diversidad, distribución y ecología del orden Plecoptera (Insecta) en Colombia, con énfasis en Anacroneuria (Perlidae). Universidad de la Amazonía. Momentos de Ciencia 7 (2): 101-112.

Zúñiga, M. del C. Rojas, A.M. \& Caicedo, G. 1993. Indicadores ambientales de calidad de agua en la cuenca del Río Cauca. Revista de la Asociación de Ingenieros Sanitarios de Antioquia- AINSA 13 (2): 17-28.

Zúñiga, M. del C. Rojas, A.M. \& Mosquera, S. 1997. Biological aspect of Ephemeroptera in rivers of southwestern Colombia (South América). Pp. 261-268. En: Landolt, P. y Sartori, M. (Eds). Ephemeroptera and Plecoptera biology, ecology and systematics. MTL, Mauron Tinguely y Lachat S.A., Switzerland.

Zúñiga, M. del C. Stark, B.P., Rojas, A.M. \& Baena, M.L. 2001. Colombian Anacroneuria (Insecta: Plecoptera: Perlidae): Biodiversity and distribution. Pp. 301-304. En: Domínguez, E. (Ed.) Trends in Research in Ephemeroptera and Plecoptera. Kluwer Academic Plenum Publishers, New York, USA.

Zúñiga, M. del C. \& Stark, B.P. 2002. New species and records of Colombian Anacroneuria (Insecta: Plecoptera: Perlidae). Spixiana 25 (3): 209-224.

Zúñiga, M. del C. Molineri, C. \& Domínguez, E. 2004. El Orden Ephemeroptera en Colombia. p. 17-41. En: Andrade. M.G., Amat García. G.,. Fernández F. (Eds.). Insectos de Colombia. Vol 3. Universidad Nacional de Colombia e Instituto Humboldt. Santafé de Bogotá, Colombia. 
Zúñiga, M. del C. Stark, B.P., Vázcones, J.J., Bersosa, F. \& Vimos, D. 2006. Colombian and Ecuadoran Anacroneuria (Plecoptera: Perlidae) - new species, records and life stages. Studies on Neotropical Fauna and Environment 41 (1): 45-57.

Zúñiga, M. del C. \& Stark, B.P. 2007. The first record of Macrogynoplax Enderlein (Plecoptera: Perlidae) from the Colombian Amazon. Illiesia 3 (11): 102-103.

Zúñiga, M. del C., Stark, B.P., Cardona, W., Tamaris, C. y Ortega, O.E. 2007. Additions to the Colombian Anacroneuria (Plecoptera: Perlidae) with descriptions of seven new species. Illiesia 3 (13): 127-149.
Zúñiga, M. del C. y Cardona, W. 2009. Bioindicadores de calidad de agua y caudal ambiental. Pp. 167-198, En: Cantera, J., Carvajal, Y. y Castro, L. (Compiladores). Caudal ambiental: conceptos experiencias y desafíos. Programa Editorial de la Universidad del Valle, Cali, Colombia.

Zúñiga, M. del C. Días, L., Martínez, D., Zabala, G. \& Bacca, T. 2009. The first record of Claudioperla Illies (Plecoptera: Gripopterygidae) from Colombia. Aquatic Insects 31 (Supplement 1): 743-744. 